ESAIM: COCV 19 (2013) 587-615

DOI: $10.1051 / \mathrm{cocv} / 2012023$
ESAIM: Control, Optimisation and Calculus of Variations

www.esaim-cocv.org

\title{
OPTIMAL CONTROL OF LINEARIZED COMPRESSIBLE NAVIER-STOKES EQUATIONS
}

\author{
Shirshendu Chowdhury ${ }^{1}$ And Mythily Ramaswamy ${ }^{1}$
}

\begin{abstract}
We study an optimal boundary control problem for the two dimensional unsteady linearized compressible Navier-Stokes equations in a rectangle. The control acts through the Dirichlet boundary condition. We first establish the existence and uniqueness of the solution for the two-dimensional unsteady linearized compressible Navier-Stokes equations in a rectangle with inhomogeneous Dirichlet boundary data, not necessarily smooth. Then, we prove the existence and uniqueness of the optimal solution over the control set. Finally we derive an optimality system from which the optimal solution can be determined.
\end{abstract}

Mathematics Subject Classification. 49J20, 49K20, 35Q30, 76N25.

Received December 9, 2011. Revised April 21, 2012.

Published online February 21, 2013.

\section{INTRODUCTION}

The Navier-Stokes equations for a viscous compressible isentropic fluid in $\Omega \subset \mathbb{R}^{N}$ is

$$
\left.\begin{array}{rl}
\frac{\partial \rho}{\partial t}(t, x)+\operatorname{div}[\rho(t, x) \boldsymbol{v}(t, x)] & =0, \\
\rho(t, x)\left[\frac{\partial \boldsymbol{v}}{\partial t}(t, x)+(\boldsymbol{v}(t, x) \cdot \nabla) \boldsymbol{v}(t, x)\right] & =-\nabla p(t, x)+\mu \triangle \boldsymbol{v}(t, x)+(\lambda+\mu) \nabla[\operatorname{div} \boldsymbol{v}(t, x)], \\
p(t, x) & =a \rho^{\gamma}(t, x), \quad t>0, x \in \Omega,
\end{array}\right\}
$$

where $\rho(t, x)$ is the density of the fluid, $\boldsymbol{v}(t, x)=\left(v_{1}(t, x), \ldots, v_{N}(t, x)\right)$ denotes the velocity vector in $\mathbb{R}^{N}$ and $p(t, x)$ denotes the pressure. Note that the second equation of (1.1) componentwise is

$$
\rho\left(\frac{\partial v_{i}}{\partial t}+\boldsymbol{v} \cdot \nabla v_{i}\right)=-\frac{\partial p}{\partial x_{i}}+\mu \triangle v_{i}+(\lambda+\mu) \frac{\partial}{\partial x_{i}}[\operatorname{div} \boldsymbol{v}], i=1,2, \ldots, N
$$

Throughout this paper, we follow this same notational convention and use bold script to denote vectors and product spaces. The viscosity coefficients $\mu, \lambda$ are assumed to be constant satisfying the following thermodynamic restrictions: $\mu>0, \lambda+\mu \geqslant 0$ and the constants $a>0, \quad \gamma>1$.

Keywords and phrases. Optimal control, linearized compressible Navier-Stokes equations, boundary control, optimality system.

1 T.I.F.R Centre for Applicable Mathematics, Post Bag No. 6503, GKVK Post Office, 560065 Bangalore, India.

shirshendu@math.tifrbng.res.in; mythily@math.tifrbng.res.in 
In this paper, we study the following system, linearized around the steady state solution $\left(q_{s}(x), \mathbf{v}_{s}(x)\right)$ of $(1.1)$ in $(0, T) \times \Omega$

$$
\begin{aligned}
& \frac{\partial \sigma}{\partial t}(t, x)+\operatorname{div}\left[\sigma(t, x) \mathbf{v}_{s}(x)\right]=-\operatorname{div}\left[q_{s}(x) \mathbf{u}(t, x)\right], \\
& \frac{\partial \mathbf{u}}{\partial t}(t, x)-\frac{\mu}{q_{s}(x)} \triangle \mathbf{u}(t, x)-\frac{(\lambda+\mu)}{q_{s}(x)} \nabla[\operatorname{div} \mathbf{u}(t, x)]+\left(\mathbf{v}_{s}(x) \cdot \nabla\right) \mathbf{u}(t, x)+(\mathbf{u}(t, x) \cdot \nabla) \mathbf{v}_{s}(x) \\
& =-a \gamma q_{s}^{\gamma-2}(x) \nabla \sigma(t, x)+\frac{\sigma(t, x)}{q_{s}(x)}\left[\mathbf{f}(x)-\left(\mathbf{v}_{s}(x) \cdot \nabla\right) \mathbf{v}_{s}(x)\right] .
\end{aligned}
$$

We consider system (1.2)-(1.3) in

$$
\Omega=\left\{x=\left(x_{1}, x_{2}\right) \in \mathbb{R}^{2}: 0<x_{1}<1,0<x_{2}<h\right\}
$$

with boundary $\partial \Omega$, consisting of three disjoint portions

$$
\Gamma_{\text {in }}=\{0\} \times(0, h), \quad \Gamma_{0}=[0,1] \times\{0, h\}, \quad \Gamma_{\text {out }}=\{1\} \times(0, h) .
$$

Let us denote

$$
\Omega_{T}=(0, T) \times \Omega ; \quad \Sigma_{T}=(0, T) \times \partial \Omega .
$$

The initial and boundary conditions are

$$
\begin{gathered}
\sigma(0, x)=\sigma_{0}(x), \quad \mathbf{u}(0, x)=\mathbf{u}_{\mathbf{0}}(x) \text { in } \Omega, \\
\sigma(t, x)=w(t, x) \text { on }(0, T) \times \Gamma_{\text {in }}, \quad \mathbf{u}(t, x)=\boldsymbol{\xi}(t, x) \text { on } \Sigma_{T},
\end{gathered}
$$

where

$$
\sigma_{0} \in L^{2}(\Omega), \mathbf{u}_{\mathbf{0}} \in \mathbf{L}^{2}(\Omega), w \in L^{2}\left(0, T ; L^{2}\left(\Gamma_{\mathrm{in}}\right)\right), \boldsymbol{\xi} \in L^{2}\left(0, T ; \mathbf{L}^{2}(\partial \Omega)\right), \mathbf{f} \in \mathbf{L}^{\infty}(\Omega) \cap \mathbf{H}^{1}(\Omega) .
$$

We assume that $\left(q_{s}(x), \mathbf{v}_{s}(x)\right)=\left(q_{s}(x), v_{s 1}(x), v_{s 2}(x)\right) \in \mathbb{R}^{3}$ satisfies the following conditions:

$$
q_{s} \in C^{2}(\bar{\Omega}), q_{s}(x)>0 \text { on } \bar{\Omega},
$$

$$
\mathbf{v}_{s} \in \mathbf{C}_{c}^{2}\left(\mathbb{R}^{2}\right), v_{s 1} \geq \alpha>0 \text { on } \overline{\Gamma_{\text {in }}} \cup \overline{\Gamma_{\text {out }}} \text { for some constant } \alpha \text { and } v_{s 2}=0 \text { on } \Gamma_{0} \text {. }
$$

We first prove that the linearized system $(1.2)-(1.5)$ has a unique solution $(\sigma, \mathbf{u})$ in $L^{2}\left(0, T ;\left[H^{1}(\Omega)\right]^{\prime}\right) \times$ $L^{2}\left(0, T ; \mathbf{L}^{2}(\Omega)\right)$ in the sense of transposition, where $\left[H^{1}(\Omega)\right]^{\prime}$ denotes the dual of $H^{1}(\Omega)$. Then we consider the following optimal control problem:

$$
(P) \quad \inf \left\{J(\sigma, \mathbf{u}, w, \boldsymbol{\xi}) \mid(w, \boldsymbol{\xi}) \in L^{2}\left(0, T ; L^{2}\left(\Gamma_{\text {in }}\right)\right) \times L^{2}\left(0, T ; \mathbf{L}^{2}(\partial \Omega)\right),(\sigma, \mathbf{u}, w, \boldsymbol{\xi}) \text { satisfies }(1.2)-(1.5)\right\},
$$

where

$$
\begin{aligned}
J(\sigma, \mathbf{u}, w, \boldsymbol{\xi})= & \frac{1}{2} \int_{0}^{T}||\left|\sigma-\sigma^{d}\right| \|_{\left[H^{1}(\Omega)\right]^{\prime}}^{2} \mathrm{~d} t+\frac{1}{2} \int_{0}^{T} \int_{\Omega}\left|\mathbf{u}-\mathbf{u}^{d}\right|^{2} \mathrm{~d} x \mathrm{~d} t \\
& +\frac{\beta}{2}\left[\int_{0}^{T} \int_{\Gamma_{\mathrm{in}}} w^{2} \mathrm{~d} \mathbf{s} \mathrm{d} t+\int_{0}^{T} \int_{\partial \Omega}|\boldsymbol{\xi}|^{2} d \mathbf{s} \mathrm{d} t\right],
\end{aligned}
$$

$\beta>0,\left(\sigma^{d}, \mathbf{u}^{d}\right)=\left(\sigma^{d}, u_{1}^{d}, u_{2}^{d}\right) \in L^{2}\left(0, T ;\left[H^{1}(\Omega)\right]^{\prime}\right) \times L^{2}\left(0, T ; \mathbf{L}^{2}(\Omega)\right)$ is the desired profile and $\||\cdot|\|_{\left[H^{1}(\Omega)\right]^{\prime}}$ is a norm in the dual of $H^{1}(\Omega)$, equivalent to the usual norm in $\left[H^{1}(\Omega)\right]^{\prime}$. It is necessary to consider this norm to get 
a well posed optimality system. We discuss this norm in Section 4 . Then we show the existence and uniqueness of the optimal solution over the control set and derive the optimality system.

In our system, the coupling between the hyperbolic character of the first order transport equation and the parabolic character of the second order linearized momentum equation leads to some difficulties mainly regarding regularity which are interesting to understand.

The main novelty here is that the boundary data are not too regular. If they are regular, one can use the lifting procedure and the standard fixed point argument in suitable function spaces for (1.2)-(1.5) to get the existence of a solution. We mention some details regarding this in Remark 3.8. But here we need to interpret the solution of (1.2)-(1.5) in the sense of transposition to get the solution $(\sigma, \mathbf{u}) \in L^{2}\left(0, T ;\left[H^{1}(\Omega)\right]^{\prime}\right) \times L^{2}\left(0, T ; \mathbf{L}^{2}(\Omega)\right)$. For this we first study the adjoint system for regular data and prove the existence of a unique solution using fixed point method.

Such a linearized system around a steady solution is also considered by Girinon [5] in $\mathbb{R}^{2}$ but with homogeneous Dirichlet boundary condition and slightly different assumptions on $q_{s}, \mathbf{v}_{s}$ and $\mathbf{f}$ :

$$
q_{s} \in C^{1}(\bar{\Omega}), q_{s}(x)>0 \text { on } \bar{\Omega}, \mathbf{v}_{s} \in \mathbf{C}_{c}^{2}\left(\mathbb{R}^{2}\right), v_{s 1}>0 \text { on } \Gamma_{\text {in }} \cup \Gamma_{\text {out }}, \mathbf{v}_{s}=\mathbf{0} \text { on } \Gamma_{0} \text { and } \mathbf{f} \in \mathbf{L}^{\infty}(\Omega) .
$$

He proved in [5], the existence and uniqueness of the solution for the linearized system. Here we consider the linearized system with nonhomogeneous Dirichlet $L^{2}$ boundary data, $\mathbf{f} \in \mathbf{L}^{\infty}(\Omega) \cap \mathbf{H}^{1}(\Omega)$ and assumptions (1.7)(1.8). The $C^{2}$ assumptions on $\left(q_{s}, \mathbf{v}_{s}\right), v_{s 1} \geq \alpha>0$ on $\overline{\Gamma_{\text {in }}} \cup \overline{\Gamma_{\text {out }}}$ and $\mathbf{f} \in \mathbf{L}^{\infty}(\Omega) \cap \mathbf{H}^{1}(\Omega)$ are used to get $H^{1}$ estimate for the solution of the transport equation in the next section.

Geymonat and Leyland study in [4] the linearized system in a bounded domain with homogeneous Dirichlet boundary conditions in $\mathbb{R}^{N}, N \geq 2$ using semigroup theory for both the transport and the Stokes part and proved the existence of a unique mild solution in $C\left([0, T] ; \mathbf{L}^{2}(\Omega)\right)$ for the full system. The space regularity that can be obtained for the transport equation using semigroup theory is not sufficient for us to get a well posed adjoint system. So we use the representation formula for the transport equation to get $H^{1}$ regularity.

Neustupa in [8] studies the linearized system in a bounded domain $\Omega$ in $\mathbb{R}^{3}$ with homogeneous Dirichlet boundary condition for velocity when the boundary of the domain is $C^{2, \alpha}, \alpha \in(0,1),\left(q_{s}, \mathbf{v}_{s}\right) \in \mathbf{C}^{3, \alpha}(\bar{\Omega})$ and $\mathbf{v}_{s} \cdot \mathbf{n}=0$ on $\partial \Omega$, where $\mathbf{n}$ denotes unit outward normal to $\partial \Omega$. Using semigroup approach, he proved the existence of a unique mild solution in $C([0, T] ; \mathcal{X})$, where $\mathcal{X}$

$$
\mathcal{X}=\left\{(\sigma, \mathbf{v}) \in C^{1, \alpha}(\bar{\Omega}) \times \mathbf{C}^{0, \alpha}(\bar{\Omega}) \mid \int_{\Omega} \sigma \mathrm{d} x=0\right\} .
$$

He used the representation formula to study the initial value problem for the transport equation. Here we study the initial boundary value problem for the transport equation and show the existence of the solution in a Sobolev space. Using the classical method of characteristics, we find the representation formula for the solution of the transport equation and prove $H^{1}$ estimate of solution. Regularity results for the initial value problem for transport equation using the representation formula, are already known. Regularity estimate for the initial boundary value problem of transport equation in regular bounded domain has also been studied by Judovič and Valli. Using the representation formula, Judovič in [7] established the existence of classical solution in a bounded domain in $\mathbb{R}^{2}$ with $C^{4}$ boundary. Valli and Zajczkowski in [11] proved existence of $H^{2}$ solution for transport equation with a lower order term in a bounded domain in $\mathbb{R}^{3}$ with $C^{2}$ boundary. To the authors knowledge, $H^{1}$ estimate of the solution for the initial boundary value problem for the transport equation in a rectangle in $\mathbb{R}^{2}$ is new and so the detailed Proof of Theorem 2.6 is one of the contributions of our work in this paper.

Raymond considered the linearized problem for incompressible Navier-Stokes equation in a bounded domain in $\mathbb{R}^{2}$ and $\mathbb{R}^{3}$ with weaker boundary data and proved the existence of the global weak solution in [9]. An optimal control problem for the linearized Boussinesq system has been studied by Raymond and Nguyen in [10] and optimality conditions are derived. In Boussinesq system Convection-Diffusion equation is coupled with the linearized incompressible Navier-Stokes equation, where both the equations are of similar nature unlike (1.2)(1.3). Our cost functional is inspired by the cost functional used by Gunzburger and Manservisi [6] for velocity tracking problem for incompressible Navier-Stokes in a bounded two-dimensional domain. 
This paper is organized as follows. In Section 2, we study the adjoint system of (1.2)-(1.5) and prove the existence and uniqueness of the solution. In Section 3, we study the existence of a unique solution for the linearized system (1.2)-(1.5) with $L^{2}$ boundary data via the transposition method. In Section 4, we establish the existence of a unique optimal control. Then optimality conditions are derived. We give a detailed Proof of Theorem 2.6 on $H^{1}$ regularity estimate and some trace result for the transport equation in Appendix A.

\section{Adjoint system}

In order to define the solution of the linearized system (1.2)-(1.5) in the sense of transposition we consider first the following adjoint system in $\Omega_{T}$ with homogeneous terminal and boundary conditions

$$
\begin{gathered}
-\frac{\partial \psi}{\partial t}(t, x)-\mathbf{v}_{s}(x) \cdot \nabla \psi(t, x)=\frac{\left[\mathbf{f}(x)-\left(\mathbf{v}_{s}(x) \cdot \nabla\right) \mathbf{v}_{s}(x)\right]}{q_{s}(x)} \cdot \boldsymbol{\phi}(t, x)+a \gamma \operatorname{div}\left[q_{s}^{\gamma-2}(x) \phi(t, x)\right]+F(t, x), \\
-\frac{\partial \phi}{\partial t}(t, x)-\mu \triangle\left[\frac{\phi(t, x)}{q_{s}(x)}\right]-(\lambda+\mu) \nabla\left\{\operatorname{div}\left[\frac{\phi(t, x)}{q_{s}(x)}\right]\right\}-\left(\operatorname{div}\left[\phi_{1}(t, x) \mathbf{v}_{s}(x)\right], \operatorname{div}\left[\phi_{2}(t, x) \mathbf{v}_{s}(x)\right]\right) \\
+\left(\nabla \mathbf{v}_{s}\right)^{T} \phi(t, x)=q_{s}(x) \nabla \psi(t, x)+\mathbf{G}(t, x), \\
\psi(T, x)=0, \phi(T, x)=\mathbf{0} \text { in } \Omega, \\
\psi(t, x)=0 \text { on }(0, T) \times \Gamma_{\text {out }}, \quad \phi(t, x)=\mathbf{0} \text { on } \Sigma_{T},
\end{gathered}
$$

where $\left(\nabla \mathbf{v}_{s}\right)^{T}$ denotes the transpose of the Jacobian matrix of $\mathbf{v}_{s}$, i.e.

$$
\left(\nabla \mathbf{v}_{s}\right)^{T}=\left(\begin{array}{ll}
\frac{\partial v_{s 1}}{\partial x_{1}} & \frac{\partial v_{s 2}}{\partial x_{1}} \\
\frac{\partial v_{s 1}}{\partial x_{2}} & \frac{\partial v_{s 2}}{\partial x_{2}}
\end{array}\right)
$$

and $(F, \mathbf{G}) \in L^{2}\left(0, T ; H^{1}(\Omega)\right) \times L^{2}\left(0, T ; \mathbf{L}^{2}(\Omega)\right)$.

For $(F, \mathbf{G}) \in L^{2}\left(0, T ; H^{1}(\Omega)\right) \times L^{2}\left(0, T ; \mathbf{L}^{2}(\Omega)\right)$, the $C^{2}$ assumptions in $(1.7)-(1.8)$ on $q_{s}, \mathbf{v}_{s}$ and $\mathbf{f} \in \mathbf{L}^{\infty}(\Omega) \cap$ $\mathbf{H}^{1}(\Omega)$ are used to conclude that the R.H.S of $(2.1)-(2.2)$ belongs to $L^{2}\left(0, T ; H^{1}(\Omega)\right) \times L^{2}\left(0, T ; \mathbf{L}^{2}(\Omega)\right)$. Using this in the next two subsections, we study the regularity of the solution of adjoint system $(2.1)-(2.4)$.

\subsection{Adjoint continuity equation}

The first equation (2.1) of the adjoint system with homogeneous terminal and boundary conditions can be written in the following form of an initial boundary value problem by defining $\breve{\psi}(t, x)=\psi(T-t, x)$. Then

$$
\left.\begin{array}{c}
\frac{\partial \check{\psi}}{\partial t}(t, x)-\mathbf{v}_{s}(x) \cdot \nabla \check{\psi}(t, x)=\varphi(t, x) \text { in } \Omega_{T}, \\
\check{\psi}(0, x)=0 \text { in } \Omega, \quad \check{\psi}(t, x)=0 \text { on }(0, T) \times \Gamma_{\text {out }},
\end{array}\right\}
$$

where (1.8) holds for $\mathbf{v}_{s}$. Our aim in this section is to find the explicit solution of (2.5) using the method of characteristics, initially for $\varphi$ smooth and later in $L^{2}\left(0, T ; H^{1}(\Omega)\right)$ and then use the representation formula to study the $H^{1}$ regularity of the solution. This will be required to show that the adjoint system (2.1)-(2.4) is well posed.

Let $(\tau, x)=\left(\tau, x_{1}, x_{2}\right)$ be any point in the cube $\Omega_{T}$. We consider the O.D.E:

$$
\frac{\mathrm{d} \mathbf{X}}{\mathrm{d} t}=-\mathbf{v}_{s}(\mathbf{X}), \quad \mathbf{X}(t, \tau, x)=x \text { for } t=\tau, t \in \mathbb{R} .
$$




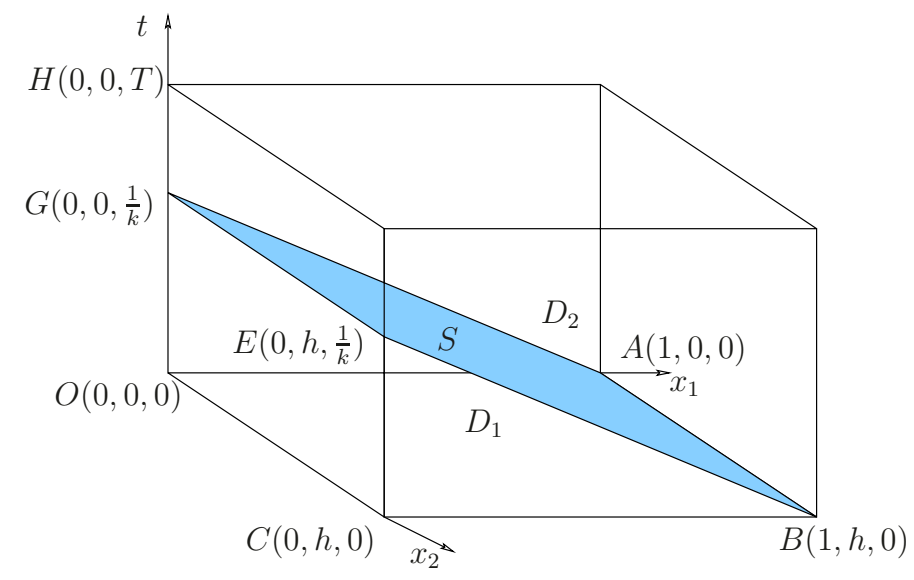

Figure 1. Partition of the cube for $\mathbf{v}_{s}=(k, 0)$.

The solution

$$
\mathbf{X}(t, \tau, x)=\left(X_{1}(t, \tau, x), X_{2}(t, \tau, x)\right)
$$

for $t<\tau$ can hit the boundary of the cube only at $t=0$ or $x_{1}=1$ plane because $\mathbf{v}_{s}$ satisfies (1.8). This leads us to the partition of the cube as follows

$$
\begin{aligned}
D_{1} & :=\left\{(\tau, x) \in \Omega_{T}: \mathbf{X}(0, \tau, x) \in \Omega\right\} \\
D_{2} & :=\left\{(\tau, x) \in \Omega_{T}: X_{1}\left(t_{2}, \tau, x\right)=1 \text { for some } t_{2}, 0<t_{2}<\tau\right\} \\
S & :=\left\{(\tau, x) \in \Omega_{T}: X_{1}(0, \tau, x)=1\right\} .
\end{aligned}
$$

Remark 2.1. If $\mathbf{v}_{s}(x)=(k, 0) \forall x \in \bar{\Omega}$, where $k>0$ is a constant, then

$$
X_{1}(t, \tau, x)=-k t+x_{1}+k \tau, X_{2}(t, \tau, x)=x_{2}, \quad t_{2}(\tau, x)=\frac{1}{k}\left(x_{1}-1\right)+\tau .
$$

See Figure 1, where $S$ denotes the interface, which is plane now and given by the equation: $x_{1}+k t=1$ for $(t, x) \in \Omega_{T}$.

Proposition 2.2. Under the assumption (1.8) on $\mathbf{v}_{s}$, there exists a function $t_{2}: D_{2} \longrightarrow \mathbb{R}$ such that $t_{2}(\tau, x)$ is a $C^{2}$ function of all the variables and

$$
\frac{\partial t_{2}}{\partial x_{i}}(\tau, x)=\frac{\frac{\partial X_{1}}{\partial x_{i}}\left(t_{2}, \tau, x\right)}{v_{s 1}\left(\mathbf{X}\left(t_{2}, \tau, x\right)\right)}, \frac{\partial t_{2}}{\partial \tau}(\tau, x)=\frac{\frac{\partial X_{1}}{\partial \tau}\left(t_{2}, \tau, x\right)}{v_{s 1}\left(\mathbf{X}\left(t_{2}, \tau, x\right)\right)}
$$

Also,

$$
\frac{\partial t_{2}}{\partial x_{i}}(\tau, x) \in L^{\infty}\left(D_{2}\right) \text { and } \frac{\partial t_{2}}{\partial \tau}(\tau, x) \in L^{\infty}\left(D_{2}\right)
$$

Proof. For each $\left(t_{0}, x_{0}\right) \in D_{2}$, the solution $\mathbf{X}\left(t, t_{0}, x_{0}\right)$ of (2.6) starting from $x_{0}$ at $t=t_{0}$, satisfies

$$
X_{1}\left(t_{2}^{0}, t_{0}, x_{0}\right)=1
$$


for some $t_{2}^{0} \in\left(0, t_{0}\right)$, by the definition of $D_{2}$. Then $v_{s 1}\left(\mathbf{X}\left(t_{2}^{0}, t_{0}, x_{0}\right)\right)>0$ as $v_{s 1}>0$ on $\{1\} \times[0, h]$ by $(1.8)$. The O.D.E (2.6) gives

$$
\frac{\mathrm{d} X_{1}}{\mathrm{~d} t}\left(t_{2}^{0}, t_{0}, x_{0}\right)<0
$$

and hence in particular nonzero.

Define the function $\mathcal{H}(t, \tau, x)=X_{1}(t, \tau, x)-1$ on $\mathbb{R} \times D_{2}$. Since $\mathbf{v}_{s}(x) \in \mathbf{C}_{c}^{2}\left(\mathbb{R}^{2}\right), X_{1}(t, \tau, x)$ is a $C^{2}$ function from $\mathbb{R} \times D_{2}$ into $\mathbb{R}$ and hence $\mathcal{H}$ is a $C^{2}$ function on $\mathbb{R} \times D_{2}$. Further

$$
\mathcal{H}\left(t_{2}^{0}, t_{0}, x_{0}\right)=0 \text { and }\left.\frac{\mathrm{d} \mathcal{H}}{\mathrm{d} t}(t, \tau, x)\right|_{\left(t_{2}^{0}, t_{0}, x_{0}\right)} \neq 0 .
$$

Applying implicit function theorem on $\mathcal{H}$ at $\left(t_{2}^{0}, t_{0}, x_{0}\right)$, there exists a 3 -dimensional open set $W$ in $D_{2}$ containing $\left(t_{0}, x_{0}\right)$ and a $C^{2}$ map $t_{2}: W \longrightarrow \mathbb{R}$ such that

$$
t_{2}\left(t_{0}, x_{0}\right)=t_{2}^{0}, \quad X_{1}\left(t_{2}(\tau, x), \tau, x\right)=1 \quad \text { for } \quad(\tau, x) \in W .
$$

After differentiation we get (2.10). Since $v_{s 1} \geq \alpha>0$ on $\{1\} \times[0, h],(2.11)$ follows from (2.10). This completes the proof.

Remark 2.3. Using the continuous dependence on initial data for the solution of ODE (2.6), we can show that $t_{2}(\tau, x)$ converges to zero when $(\tau, x) \in D_{2}$, converges to a point in $\mathrm{S}$. It helps in the next proposition to conclude that solution of $(2.5)$ on $D_{1}$ and $D_{2}$ matches on $S$.

Proposition 2.4. Under assumption (1.8) on $\mathbf{v}_{s}$, for $\varphi \in C_{c}^{\infty}\left(0, T ; C^{\infty}(\bar{\Omega})\right)$ equation $(2.5)$ has a strong solution (i.e. satisfying the equation almost everywhere) $\check{\psi} \in C([0, T] ; C(\bar{\Omega}))$ and in fact $\check{\psi}$ is $C^{1}\left(D_{1} \cup D_{2}\right)$.

Proof. Using (2.6) and the first equation of (2.5) for $t<\tau$,

$$
\frac{\mathrm{d}}{\mathrm{d} t}\{\check{\psi}(t, \mathbf{X}(t, \tau, x))\}=\varphi(t, \mathbf{X}(t, \tau, x)), \quad(\tau, x) \in \Omega_{T} .
$$

Integrating this between $T_{1}$ and $T_{2}$ for $0 \leq T_{1} \leq T_{2} \leq \tau$, we get

$$
\check{\psi}\left(T_{2}, \mathbf{X}\left(T_{2}, \tau, x\right)\right)-\check{\psi}\left(T_{1}, \mathbf{X}\left(T_{1}, \tau, x\right)\right)=\int_{T_{1}}^{T_{2}} \varphi(s, \mathbf{X}(s, \tau, x)) \mathrm{d} s .
$$

Case 1. Let $(\tau, x) \in D_{1} \cup S$. Choosing $T_{1}=0, T_{2}=\tau$ in (2.12) and using the second equation of (2.5) we get,

$$
\check{\psi}(\tau, x)=\int_{0}^{\tau} \varphi(s, \mathbf{X}(s, \tau, x)) \mathrm{d} s .
$$

Case 2. Let $(\tau, x) \in D_{2}$. Then choosing $T_{1}=t_{2}(\tau, x)$, the time when the trajectory $\mathbf{X}(t, \tau, x)$ hits $x_{1}=1$ plane, $T_{2}=\tau$ in (2.12) and using the second equation of (2.5) we get,

$$
\check{\psi}(\tau, x)=\int_{t_{2}(\tau, x)}^{\tau} \varphi(s, \mathbf{X}(s, \tau, x)) \mathrm{d} s .
$$

Combining both, the solution of (2.5) can be written as

$$
\check{\psi}(t, x)=\left\{\int_{0}^{t} \varphi(s, \mathbf{X}(s, t, x)) \mathrm{d} s\right\} \chi_{D_{1} \cup S}+\left\{\int_{t_{2}(t, x)}^{t} \varphi(s, \mathbf{X}(s, t, x)) \mathrm{d} s\right\} \chi_{D_{2}} .
$$


$\mathbf{X}(s, t, x)$ is $C^{2}$ on $\mathbb{R} \times \mathbb{R} \times \mathbb{R}^{2}$. Also $\varphi$ and $t_{2}$ are $C^{2}$ functions for all the variables on $\overline{\Omega_{T}}$ and $D_{2}$ (using Prop. 2.2) respectively. Therefore from (2.13), using Remark 2.3, we get $\check{\psi}$ is at least a continuous function of $(t, x)$ on $\overline{\Omega_{T}}$.

Now let us calculate the derivatives of $\check{\psi}$ with respect to space and time. From (2.13) we get for $i=1,2$ and $(t, x) \in D_{1} \cup S$

$$
\frac{\partial \check{\psi}}{\partial x_{i}}(t, x)=\int_{0}^{t} \frac{\partial}{\partial x_{i}}\{\varphi(s, \mathbf{X}(s, t, x))\} \mathrm{d} s, \quad \frac{\partial \breve{\psi}}{\partial t}(t, x)=\int_{0}^{t} \frac{\partial}{\partial t}\{\varphi(s, \mathbf{X}(s, t, x))\} \mathrm{d} s+\varphi(t, x) .
$$

For $(t, x) \in D_{2}$, since $X_{1}\left(t_{2}, t, x\right)=1$,

$$
\begin{array}{r}
\frac{\partial \check{\psi}}{\partial x_{i}}(t, x)=\int_{t_{2}(t, x)}^{t} \frac{\partial}{\partial x_{i}}\{\varphi(s, \mathbf{X}(s, t, x))\} \mathrm{d} s-\frac{\partial t_{2}}{\partial x_{i}}(t, x) \varphi\left(t_{2}(t, x), 1, X_{2}\left(t_{2}(t, x), t, x\right)\right), \\
\frac{\partial \check{\psi}}{\partial t}(t, x)=\int_{t_{2}(t, x)}^{t} \frac{\partial}{\partial t}\{\varphi(s, \mathbf{X}(s, t, x))\} \mathrm{d} s+\varphi(t, x)-\frac{\partial t_{2}}{\partial t}(t, x) \varphi\left(t_{2}(t, x), 1, X_{2}\left(t_{2}(t, x), t, x\right)\right) .
\end{array}
$$

Hence from (2.14)-(2.16) we get $\left.\check{\psi}\right|_{D_{1}},\left.\check{\psi}\right|_{D_{2}}$ are $C^{1}$ functions with respect $t, x$ on $D_{1}$ and $D_{2}$ respectively.

Remark 2.5. The hitting time $t_{2}(\tau, x)$ will satisfy the following equation on $D_{2}$

$$
\frac{\partial t_{2}}{\partial \tau}(\tau, x)-\mathbf{v}_{s}(x) \cdot \nabla t_{2}(\tau, x)=0 .
$$

Using this equation (2.17), we can see that the representation formula (2.13) is indeed a solution of equation (2.5) after differentiation.

Proposition 2.4 leads to the following $H^{1}$ regularity result for $\check{\psi}$.

Theorem 2.6. If $\varphi \in L^{2}\left(0, T ; H^{1}(\Omega)\right)$, then equation (2.5) has a unique strong solution $\check{\psi} \in L^{\infty}\left(0, T ; H^{1}(\Omega)\right) \cap$ $H^{1}\left(0, T ; L^{2}(\Omega)\right)$ and we have the following estimate:

$$
\max _{[0, T]}\|\check{\psi}(t)\|_{H^{1}(\Omega)}+\left\|\frac{\partial \check{\psi}}{\partial t}\right\|_{L^{2}\left(0, T ; L^{2}(\Omega)\right)} \leq C\left(\mathbf{v}_{s}, T, \Omega\right)\|\varphi\|_{L^{2}\left(0, T ; H^{1}(\Omega)\right)}
$$

for some constant $C\left(\mathbf{v}_{s}, T, \Omega\right)$. In fact $\check{\psi} \in C\left([0, T] ; H^{1}(\Omega)\right) \cap H^{1}\left(0, T ; L^{2}(\Omega)\right)$ and solution is unique in the class $L^{2}\left(0, T ; H^{1}(\Omega)\right) \cap H^{1}\left(0, T ; L^{2}(\Omega)\right)$.

Details of the $L^{2}$ integrability of the derivatives of $\check{\psi}$ and uniqueness of $\check{\psi}$ required for the proof are given in the appendix of this paper. This will be required in Section 2.3 to show that the adjoint system (2.1)-(2.4) is well posed.

\subsection{Adjoint linearized momentum equation}

Let $B$ be the operator defined in $\mathbf{L}^{2}(\Omega)$ by

$$
D(B)=\mathbf{H}^{2}(\Omega) \cap \mathbf{H}_{0}^{1}(\Omega), B \mathbf{u}=-\frac{\mu}{q_{s}} \triangle \mathbf{u}-\frac{(\lambda+\mu)}{q_{s}} \nabla(\operatorname{div} \mathbf{u})+\left(\mathbf{v}_{s} \cdot \nabla\right) \mathbf{u}+(\mathbf{u} \cdot \nabla) \mathbf{v}_{s}
$$

In this section, we study the following system with homogeneous terminal and boundary condition:

$$
\left.\begin{array}{c}
-\frac{\partial \phi}{\partial t}(t, x)+B^{*} \phi(t, x)=\boldsymbol{\Upsilon}(t, x) \text { in } \Omega_{T} \\
\phi(T, x)=\mathbf{0} \text { in } \Omega, \quad \phi(t, x)=\mathbf{0} \text { on } \Sigma_{T}
\end{array}\right\}
$$


where $\boldsymbol{\Upsilon} \in L^{2}\left(0, T ; \mathbf{L}^{2}(\Omega)\right)$ and $B^{*} \boldsymbol{\phi}=\left(\left(B^{*} \phi\right)_{1},\left(B^{*} \phi\right)_{2}\right)$ is the adjoint of $B$ from $D\left(B^{*}\right)=\mathbf{H}^{2}(\Omega) \cap \mathbf{H}_{0}^{1}(\Omega) \subset$ $\mathbf{L}^{2}(\Omega)$ into $\mathbf{L}^{2}(\Omega)$ defined as:

$$
B^{*} \phi=-\mu \triangle\left(\frac{\phi}{q_{s}}\right)-(\lambda+\mu) \nabla\left[\operatorname{div}\left(\frac{\phi}{q_{s}}\right)\right]-\left(\operatorname{div}\left(\phi_{1} \mathbf{v}_{s}\right), \operatorname{div}\left(\phi_{2} \mathbf{v}_{s}\right)\right)+\left(\nabla \mathbf{v}_{s}\right)^{T} \boldsymbol{\phi}
$$

for $\phi=\left(\phi_{1}, \phi_{2}\right) \in \mathbf{H}^{2}(\Omega) \cap \mathbf{H}_{0}^{1}(\Omega)$.

We look for a weak solution of equation (2.20) in the following sense:

Definition 2.7. A function $\phi \in L^{2}\left(0, T ; \mathbf{L}^{2}(\Omega)\right)$ is a weak solution of problem (2.20) if for all $\zeta$ in $D(B)$, $(\zeta, \phi(t))_{\mathbf{L}^{2}(\Omega)}$ belongs to $H^{1}(0, T)$ and

$$
\begin{aligned}
\frac{\mathrm{d}}{\mathrm{d} t}(\zeta,-\phi(t))_{\mathbf{L}^{2}(\Omega)} & =(-B \zeta, \phi(t))_{\mathbf{L}^{2}(\Omega)}+(\zeta, \boldsymbol{\Upsilon}(t))_{\mathbf{L}^{2}(\Omega)}, \\
(\zeta, \phi(T))_{\mathbf{L}^{2}(\Omega)} & =0
\end{aligned}
$$

for almost all $\mathrm{t}$ in $[0, \mathrm{~T}]$.

Let us define the bilinear form $b$ on $\mathbf{H}_{0}^{1}(\Omega) \times \mathbf{H}_{0}^{1}(\Omega)$ associated with the operator $B^{*}$ as:

$$
b(\boldsymbol{\Phi}, \boldsymbol{\Psi})=\mu \int_{\Omega}\left[\nabla \boldsymbol{\Psi}: \nabla\left(\frac{\boldsymbol{\Phi}}{q_{s}}\right)\right] \mathrm{d} x+(\lambda+\mu) \int_{\Omega} \operatorname{div} \boldsymbol{\Psi} \operatorname{div}\left(\frac{\boldsymbol{\Phi}}{q_{s}}\right) \mathrm{d} x+\int_{\Omega}\left[(\boldsymbol{\Psi} \cdot \nabla) \mathbf{v}_{s} \cdot \boldsymbol{\Phi}+\left(\mathbf{v}_{s} \cdot \nabla\right) \boldsymbol{\Psi} \cdot \boldsymbol{\Phi}\right] \mathrm{d} x,
$$

where

$$
\left[\nabla \boldsymbol{\Psi}: \nabla\left(\frac{\boldsymbol{\Phi}}{q_{s}}\right)\right]=\sum_{i=1}^{2}\left(\nabla \Psi_{i}\right) \cdot \nabla\left(\frac{\Phi_{i}}{q_{s}}\right),(\boldsymbol{\Psi} \cdot \nabla) \mathbf{v}_{s} \cdot \boldsymbol{\Phi}=\sum_{i=1}^{2}\left(\boldsymbol{\Psi} \cdot \nabla v_{s i}\right) \Phi_{i}, \quad\left(\mathbf{v}_{s} \cdot \nabla\right) \boldsymbol{\Psi} \cdot \boldsymbol{\Phi}=\sum_{i=1}^{2}\left(\mathbf{v}_{s} \cdot \nabla \Psi_{i}\right) \Phi_{i} .
$$

Clearly b is a continuous bilinear form on $\mathbf{H}_{0}^{1}(\Omega) \times \mathbf{H}_{0}^{1}(\Omega)$ and we can show that there exists $\lambda_{0}>0$ and $\alpha>0$ such that

$$
b(\phi, \phi)+\lambda_{0}\|\phi\|_{\mathbf{L}^{2}(\Omega)}^{2} \geq \alpha\|\phi\|_{\mathbf{H}_{0}^{1}(\Omega)}^{2} \forall \boldsymbol{\phi} \in \mathbf{H}_{0}^{1}(\Omega) .
$$

Hence using Proposition 3 (Chap. XVII, Sect. 6) of Dautray and Lions [2] we get the following result.

Proposition 2.8. $-B^{*}$ generates an analytic semigroup $S_{-B^{*}}(t)$ on $\mathbf{L}^{2}(\Omega)$ with domain $D\left(-B^{*}\right)=\mathbf{H}^{2}(\Omega) \cap$ $\mathbf{H}_{0}^{1}(\Omega)$.

Notice that defining $\boldsymbol{\eta}(t, x)=\boldsymbol{\phi}(T-t, x)$, we can write (2.20) as the following system for $\boldsymbol{\eta}$ with initial condition

$$
\left.\begin{array}{r}
\frac{\partial \boldsymbol{\eta}}{\partial t}(t, x)+B^{*} \boldsymbol{\eta}(t, x)=\boldsymbol{\Upsilon}(T-t, x) \text { in } \Omega_{T} \\
\boldsymbol{\eta}(0, x)=\mathbf{0} \text { in } \Omega, \quad \boldsymbol{\eta}(t, x)=\mathbf{0} \text { on } \Sigma_{T}
\end{array}\right\}
$$

Using the following theorem, we get the existence and regularity of the weak solution for the system (2.21). Proof can be found in the book "Representation and Control of Infinite Dimensional Systems" [1] (Prop. 3.7 in Sect. 3.6 of Part II, Chap. 1).

Theorem 2.9. Let $A$ be the infinitesimal generator of a strongly continuous analytic semigroup $\left\{S_{A}(t)\right\}_{t \geqslant 0}$ defined on a domain $D(A)$ in the Hilbert space $Z$. Then for any $T>0$ and $f \in L^{2}(0, T ; Z)$, the Cauchy problem

$$
z^{\prime}(t)=A z(t)+f(t), t \in[0, T], \quad z(0)=0 \in Z
$$

admits a unique weak solution

$$
z(t)=\int_{0}^{t}\left\langle S_{A}(t-s), f(s)\right\rangle \mathrm{d} s
$$

This $z$ in fact lies in $L^{2}(0, T ; D(A)) \cap H^{1}(0, T ; Z)$ and hence is a strong solution of the Cauchy problem. 
Thus equation (2.21) has a unique strong solution

$$
\boldsymbol{\eta}(t)=\int_{0}^{t}\left\langle S_{-B^{*}}(t-s), \boldsymbol{\Upsilon}(T-s, x)\right\rangle \mathrm{d} s
$$

and hence using the change of variable $\tau=T-s$ and $l=T-t$ we get,

$$
\phi(l)=\int_{l}^{T}\left\langle S_{-B^{*}}(\tau-l), \boldsymbol{\Upsilon}(\tau, x)\right\rangle \mathrm{d} \tau
$$

Thus for $\boldsymbol{\Upsilon} \in L^{2}\left(0, T ; \mathbf{L}^{2}(\Omega)\right)$, equation (2.20) has a unique strong solution $\boldsymbol{\phi}$ in $H^{1}\left(0, T ; \mathbf{L}^{2}(\Omega)\right)$ $\cap L^{2}\left(0, T ; \mathbf{H}^{2}(\Omega) \cap \mathbf{H}_{0}^{1}(\Omega)\right)$.

\subsection{Solution for the adjoint system}

In this section we consider the adjoint system (2.1)-(2.4) and we will show the existence and uniqueness of the strong solution by a fixed point argument. For that we need to set up a map $\Pi$ from a suitable function space into itself.

Let $\mathcal{F}: H^{1}(\Omega) \longrightarrow L^{2}\left(0, T ; \mathbf{L}^{2}(\Omega)\right)$ be defined for $\mathbf{G} \in L^{2}\left(0, T ; \mathbf{L}^{2}(\Omega)\right)$ and $q \in H^{1}(\Omega)$

$$
\mathcal{F}(q)=q_{s} \nabla q+\mathbf{G} .
$$

We want to show that for any $(F, \mathbf{G}) \in L^{2}\left(0, T ; H^{1}(\Omega)\right) \times L^{2}\left(0, T ; \mathbf{L}^{2}(\Omega)\right)$, the coupled system:

$$
\left.\begin{array}{r}
-\frac{\partial \psi}{\partial t}-\mathbf{v}_{s} \cdot \nabla \psi=\frac{\left[\mathbf{f}-\left(\mathbf{v}_{s} \cdot \nabla\right) \mathbf{v}_{s}\right]}{q_{s}} \cdot \phi+a \gamma \operatorname{div}\left(q_{s}^{\gamma-2} \phi\right)+F \text { in } \Omega_{T}, \\
\psi(T, x)=0 \text { in } \Omega, \quad \psi(t, x)=0 \text { on }(0, T) \times \Gamma_{\text {out }},
\end{array}\right\}
$$

admits a unique strong solution $(\psi, \boldsymbol{\phi})$ in $\left[H^{1}\left(0, T ; L^{2}(\Omega)\right) \cap L^{2}\left(0, T ; H^{1}(\Omega)\right)\right] \times\left[H^{1}\left(0, T ; \mathbf{L}^{2}(\Omega)\right)\right.$

$\left.\cap L^{2}\left(0, T ; \mathbf{H}^{2}(\Omega) \cap \mathbf{H}_{0}^{1}(\Omega)\right)\right]$.

Let $0<T_{1} \leq T$. For $y \in L^{2}\left(0, T_{1} ; H^{1}(\Omega)\right)$, define $\phi^{y}$ as the solution in $H^{1}\left(0, T_{1} ; \mathbf{L}^{2}(\Omega)\right) \cap L^{2}\left(0, T_{1} ; \mathbf{H}^{2}(\Omega) \cap\right.$ $\left.\mathbf{H}_{0}^{1}(\Omega)\right)$ for the equation

$$
\left.\begin{array}{rl}
-\frac{\partial \phi}{\partial t}+B^{*} \phi & =\mathcal{F}(y) \text { in } \Omega_{T_{1}}, \\
\text { in } \Omega, \quad \phi(t, x) & =\mathbf{0} \text { on } \Sigma_{T_{1}},
\end{array}\right\}
$$

given by Theorem 2.9. For this $\phi^{y}$,

$$
\left(\frac{\left[\mathbf{f}-\left(\mathbf{v}_{s} \cdot \nabla\right) \mathbf{v}_{s}\right]}{q_{s}} \cdot \phi^{y}+a \gamma \operatorname{div}\left(q_{s}^{\gamma-2} \phi^{y}\right)+F\right) \in L^{2}\left(0, T_{1} ; H^{1}(\Omega)\right) .
$$

Let $\psi^{y} \in L^{2}\left(0, T_{1} ; H^{1}(\Omega)\right)$ denote the solution of the equation

$$
\left.\begin{array}{rl}
-\frac{\partial \psi}{\partial t}-\mathbf{v}_{s} \cdot \nabla \psi & =\frac{\left[\mathbf{f}-\left(\mathbf{v}_{s} \cdot \nabla\right) \mathbf{v}_{s}\right]}{q_{s}} \cdot \phi^{y}+a \gamma \operatorname{div}\left(q_{s}^{\gamma-2} \phi^{y}\right)+F \text { in } \Omega_{T_{1}}, \\
\psi\left(T_{1}, x\right) & =0 \text { in } \Omega, \quad \psi(t, x)=0 \text { on }\left(0, T_{1}\right) \times \Gamma_{\text {out }} .
\end{array}\right\}
$$

Theorem 2.6 gives the existence of the $\psi^{y}$ in $L^{2}\left(0, T_{1} ; H^{1}(\Omega)\right)$. 
Now we define a map $\Pi$ from $L^{2}\left(0, T_{1} ; H^{1}(\Omega)\right)$ into itself by

$$
\Pi(y)=\psi^{y} .
$$

We want to show that $\Pi$ is a contraction for small $T_{1}$. For that we adapt the proof of Girinon to the case of the adjoint system. However, we give the details since there are some major differences:

(i) We work in more regular spaces as we need more regularity for the solution of the adjoint system so as to define the solution of the original system by transposition. In fact, Girinon gets a contraction map in $L^{2}\left(0, T_{1}, L^{2}(\Omega)\right)$ for $(1.2)-(1.5)$ with homogeneous boundary conditions whereas we get contraction in $L^{2}\left(0, T_{1}, H^{1}(\Omega)\right)$ for adjoint system $(2.1)-(2.4)$.

(ii) For the adjoint continuity equation we use explicit expression of the solution via method of characteristics because we need $H^{1}$ regularity of the solution while he uses the semigroup approach.

(iii) We use the semigroup approach to study the adjoint linearized momentum equation while Girinon studies the linearized momentum equation by the variational method (Galerkin method). Thus using the method of Girinon, we can get only a weak solution for adjoint continuity equation by semigroup and a weak solution for adjoint linearized momentum equation using Galerkin method. But our approach gives strong solutions for both the equations.

Proposition 2.10. There exists a natural number $N$ depending on $T, q_{s}$ and $\mathbf{v}_{s}$ such that for $T_{1}=\frac{T}{N}$, $\Pi$ is a contraction on $L^{2}\left(0, T_{1} ; H^{1}(\Omega)\right)$.

Proof. Let $y_{i} \in L^{2}\left(0, T_{1} ; H^{1}(\Omega)\right)$ for $i=1,2$ and $\phi^{i}=\phi^{y_{i}}, \psi^{i}=\psi^{y_{i}}$ be the solution of (2.24) and (2.25) corresponding to $y_{i}$ for $i=1,2$. So $\left(\phi^{\mathbf{1}}-\boldsymbol{\phi}^{\mathbf{2}}\right)$ is the solution of

$$
\left.\begin{array}{rl}
-\frac{\partial \phi}{\partial t}+B^{*} \phi & =q_{s} \nabla\left(y_{1}-y_{2}\right) \text { in } \Omega_{T_{1}}, \\
\phi\left(T_{1}, x\right) & =\mathbf{0} \text { in } \Omega, \quad \phi(t, x)=\mathbf{0} \text { on } \Sigma_{T_{1}} .
\end{array}\right\}
$$

Hence using Theorem 2.9, for $t \in\left[0, T_{1}\right]$

$$
\left\|\boldsymbol{\phi}^{\mathbf{1}}-\boldsymbol{\phi}^{\mathbf{2}}\right\|_{L^{2}\left(0, T_{1} ; \mathbf{H}^{2}(\Omega)\right)} \leq C_{3}\left\|q_{s} \nabla\left(y_{1}-y_{2}\right)\right\|_{L^{2}\left(0, T_{1} ; \mathbf{L}^{2}(\Omega)\right)} \leq C_{4}\left(q_{s}, \mathbf{v}_{s}, T\right)\left\|y_{1}-y_{2}\right\|_{L^{2}\left(0, T_{1} ; H^{1}(\Omega)\right)} .
$$

Also $\left(\psi^{1}-\psi^{2}\right)$ is the solution of

$$
\left.\begin{array}{rl}
-\frac{\partial \psi}{\partial t}-\mathbf{v}_{s} \cdot \nabla \psi & =\frac{\left[\mathbf{f}-\left(\mathbf{v}_{s} \cdot \nabla\right) \mathbf{v}_{s}\right]}{q_{s}} \cdot\left(\phi^{1}-\phi^{2}\right)+a \gamma \operatorname{div}\left[q_{s}^{\gamma-2}\left(\phi^{1}-\phi^{2}\right)\right] \text { in } \Omega_{T_{1}}, \\
\psi\left(T_{1}, x\right) & =0 \text { on } \Omega, \quad \psi(t, x)=0 \text { on }\left(0, T_{1}\right) \times \Gamma_{\text {out }} .
\end{array}\right\}
$$

Therefore using (2.18) of Theorem 2.6 we get for $t \in\left[0, T_{1}\right]$

$$
\left\|\psi^{1}(t)-\psi^{2}(t)\right\|_{H^{1}(\Omega)} \leq C\left(\mathbf{v}_{s}, T, \Omega\right)\left\|\frac{\left[\mathbf{f}-\left(\mathbf{v}_{s} \cdot \nabla\right) \mathbf{v}_{s}\right]}{q_{s}} \cdot\left(\phi^{1}-\phi^{2}\right)+\operatorname{a\gamma div}\left[q_{s}^{\gamma-2}\left(\phi^{1}-\phi^{2}\right)\right]\right\|_{L^{2}\left(0, T_{1} ; H^{1}(\Omega)\right)}
$$

and hence using (2.26)

$$
\left\|\psi^{1}(t)-\psi^{2}(t)\right\|_{H^{1}(\Omega)} \leq C_{5}\left\|\phi^{1}-\phi^{2}\right\|_{L^{2}\left(0, T_{1} ; \mathbf{H}^{2}(\Omega)\right)} \leq C_{6}\left(\mathbf{v}_{s}, T, q_{s}, \Omega, \mathbf{f}\right)\left\|y_{1}-y_{2}\right\|_{L^{2}\left(0, T_{1} ; H^{1}(\Omega)\right)} .
$$

Thus

$$
\left\|\Pi\left(y_{1}\right)-\Pi\left(y_{2}\right)\right\|_{L^{2}\left(0, T_{1} ; H^{1}(\Omega)\right)} \leq C_{6}\left(\mathbf{v}_{s}, T, q_{s}, \Omega, \mathbf{f}\right) \sqrt{T_{1}}\left\|y_{1}-y_{2}\right\|_{L^{2}\left(0, T_{1} ; H^{1}(\Omega)\right)} .
$$

Consequently $\Pi$ is a contraction for $T_{1}<\frac{1}{C_{6}\left(\mathbf{v}_{s}, T, q_{s}, \Omega, \mathbf{f}\right)^{2}}$. Therefore if we choose a natural number $N>$ $T C_{6}\left(\mathbf{v}_{s}, T, q_{s}, \Omega, \mathbf{f}\right)^{2}$, then for $T_{1}=\frac{T}{N}, \Pi$ is a contraction on $L^{2}\left(0, T_{1} ; H^{1}(\Omega)\right)$.

Hence we have the following theorem for local existence of a solution. 
Theorem 2.11. Under assumptions (1.7)-(1.8), $\mathbf{f} \in \mathbf{L}^{\infty}(\Omega) \cap \mathbf{H}^{1}(\Omega)$ and for $(F, \mathbf{G}) \in L^{2}\left(0, T ; H^{1}(\Omega)\right) \times$ $L^{2}\left(0, T ; \mathbf{L}^{2}(\Omega)\right)$, there exists $T_{1}>0$ depending on $\mathbf{v}_{s}, T, q_{s}, \Omega, \mathbf{f}$ as in the above proposition such that, the adjoint system $(2.1)-(2.4)$ has a unique strong solution $(\bar{\psi}, \bar{\phi})$ in $\left[L^{2}\left(0, T_{1} ; H^{1}(\Omega)\right) \cap H^{1}\left(0, T_{1} ; L^{2}(\Omega)\right)\right] \times$ $\left[L^{2}\left(0, T_{1} ; \mathbf{H}^{2}(\Omega) \cap \mathbf{H}_{0}^{1}(\Omega)\right) \cap H^{1}\left(0, T_{1} ; \mathbf{L}^{2}(\Omega)\right)\right]$.

It is standard to pass from local to global existence by subdividing $[0, T]$ for $T>T_{1}$, into $N$ subintervals and getting the existence in each $\left[\frac{k T}{N}, \frac{(k+1) T}{N}\right]$ using Theorem 2.11. Hence we have the following theorem for global existence of a solution. See for details, for example, the thesis of Girinon (Chap. IV, Sect. 4.3) [5].

Theorem 2.12. The adjoint system (2.1)-(2.4) admits a unique strong solution on $(0, T)$.

Remark 2.13. The solution map $(F, \mathbf{G}) \longmapsto(\psi, \phi)$ is continuous from $L^{2}\left(0, T ; H^{1}(\Omega)\right) \times L^{2}\left(0, T ; \mathbf{L}^{2}(\Omega)\right)$ into $L^{2}\left(0, T ; H^{1}(\Omega)\right) \times L^{2}\left(0, T ; \mathbf{H}^{2}(\Omega) \cap \mathbf{H}_{0}^{1}(\Omega)\right)$ using closed graph theorem.

Remark 2.14. Note that if we choose $F \in L^{2}\left(0, T ; L^{2}(\Omega)\right)$ and $\mathbf{G} \in L^{2}\left(0, T ; \mathbf{L}^{2}(\Omega)\right)$, then using Girinon [5] we already know that the adjoint system $(2.1)-(2.4)$ has a unique weak solution $(\psi, \phi)$ in $C\left([0, T] ; L^{2}(\Omega)\right) \times$ $\left[L^{2}\left(0, T ; \mathbf{H}_{0}^{1}(\Omega)\right) \cap C\left([0, T] ; \mathbf{L}^{2}(\Omega)\right)\right]$. In our set up we need $H^{1}$ regularity of $\psi$. So we work with $F \in$ $L^{2}\left(0, T ; H^{1}(\Omega)\right)$.

\section{Solution By tRAnSPOSition FOR THE LineARIZED SYSTEM}

In this section we prove the existence of a unique solution in the sense of transposition of system (1.2)-(1.3) with inhomogeneous initial and boundary conditions (1.4)-(1.5) using the adjoint system (2.1)-(2.4) and obtain continuity estimate of the solution.

Definition 3.1. A function $(\sigma, \mathbf{u}) \in L^{2}\left(0, T ;\left[H^{1}(\Omega)\right]^{\prime}\right) \times L^{2}\left(0, T ; \mathbf{L}^{2}(\Omega)\right)$ is a solution to the system (1.2)-(1.5) if for every $(F, \mathbf{G}) \in L^{2}\left(0, T ; H^{1}(\Omega)\right) \times L^{2}\left(0, T ; \mathbf{L}^{2}(\Omega)\right)$,

$$
\begin{aligned}
\int_{0}^{T}\langle\sigma, F\rangle_{\left(\left[H^{1}(\Omega)\right]^{\prime}, H^{1}(\Omega)\right)} \mathrm{d} t+\int_{0}^{T} \int_{\Omega} \mathbf{G} \cdot \mathbf{u} \mathrm{d} x \mathrm{~d} t & =\int_{\Omega} \sigma_{0} \psi(0, x) \mathrm{d} x+\int_{\Omega} \mathbf{u}_{0} \cdot \boldsymbol{\phi}(0, x) \mathrm{d} x+\int_{0}^{T} \int_{\Gamma_{\mathrm{in}}} w \psi v_{s 1} \mathrm{~d} \mathbf{s} \mathrm{d} t \\
& -\int_{0}^{T} \int_{\partial \Omega}\left\{\mu\left[\frac{\partial}{\partial \mathbf{n}}\left(\frac{\phi}{q_{s}}\right)\right]+(\lambda+\mu)\left[\operatorname{div}\left(\frac{\phi}{q_{s}}\right)\right] \mathbf{n}\right\} \cdot \boldsymbol{\xi} \mathrm{d} \mathbf{s} \mathrm{d} t,
\end{aligned}
$$

where $(\psi, \boldsymbol{\phi})$ is the strong solution to the adjoint system $(2.1)-(2.4)$ with this $(F, \mathbf{G}) \in L^{2}\left(0, T ; H^{1}(\Omega)\right) \times$ $L^{2}\left(0, T ; \mathbf{L}^{2}(\Omega)\right)$.

Notice that the term $\int_{0}^{T} \int_{\partial \Omega}\left\{\mu\left[\frac{\partial}{\partial \mathbf{n}}\left(\frac{\phi}{q_{s}}\right)\right]+(\lambda+\mu)\left[\operatorname{div}\left(\frac{\phi}{q_{s}}\right)\right] \mathbf{n}\right\} \cdot \boldsymbol{\xi} \mathrm{d} \mathbf{s} \mathrm{d} t$ denotes

$$
\sum_{i=1}^{2} \int_{0}^{T} \int_{\partial \Omega}\left\{\mu\left[\nabla\left(\frac{\phi_{i}}{q_{s}}\right) \cdot \mathbf{n}\right]+(\lambda+\mu)\left[\operatorname{div}\left(\frac{\phi}{q_{s}}\right)\right] n_{i}\right\} \xi_{i} \mathrm{~d} \mathbf{s} \mathrm{d} t .
$$

We first consider system (1.2)-(1.3) with homogeneous initial condition in $\Omega_{T}$ and inhomogeneous boundary conditions namely, (1.2), (1.3) with

$$
\begin{gathered}
\sigma(0, x)=0, \mathbf{u}(0, x)=\mathbf{0} \text { in } \Omega \\
\sigma(t, x)=w(t, x) \text { on }(0, T) \times \Gamma_{\text {in }}, \quad \mathbf{u}(t, x)=\boldsymbol{\xi}(t, x) \text { on } \Sigma_{T} .
\end{gathered}
$$

Now we show that the system $\{(1.2),(1.3),(3.1),(3.2)\}$ is well posed. 
Theorem 3.2. For every $(w, \boldsymbol{\xi}) \in L^{2}\left(0, T ; L^{2}\left(\Gamma_{\mathrm{in}}\right)\right) \times L^{2}\left(0, T ; \mathbf{L}^{2}(\partial \Omega)\right)$, the system $\{(1.2),(1.3),(3.1),(3.2)\}$ admits a unique solution $(\hat{\sigma}, \hat{\mathbf{u}}) \in L^{2}\left(0, T ;\left[H^{1}(\Omega)\right]^{\prime}\right) \times L^{2}\left(0, T ; \mathbf{L}^{2}(\Omega)\right)$ in the sense of transposition and the operator

$$
(w, \boldsymbol{\xi}) \longrightarrow(\hat{\sigma}, \hat{\mathbf{u}})
$$

is linear and continuous from $L^{2}\left(0, T ; L^{2}\left(\Gamma_{\mathrm{in}}\right)\right) \times L^{2}\left(0, T ; \mathbf{L}^{2}(\partial \Omega)\right)$ into $L^{2}\left(0, T ;\left[H^{1}(\Omega)\right]^{\prime}\right) \times L^{2}\left(0, T ; \mathbf{L}^{2}(\Omega)\right)$.

Proof.

\section{Uniqueness:}

If $(w, \boldsymbol{\xi})=(0, \mathbf{0})$, we have

$$
\int_{0}^{T}\langle\sigma, F\rangle_{\left(\left[H^{1}(\Omega)\right]^{\prime}, H^{1}(\Omega)\right)} \mathrm{d} t+\int_{0}^{T} \int_{\Omega} \mathbf{G} \cdot \mathbf{u} \mathrm{d} x \mathrm{~d} t=0
$$

for all $(F, \mathbf{G}) \in L^{2}\left(0, T ; H^{1}(\Omega)\right) \times L^{2}\left(0, T ; \mathbf{L}^{2}(\Omega)\right)$. Thus $(\sigma, \mathbf{u})=(0, \mathbf{0})$ and so the solution to system $\{(1.2),(1.3),(3.1),(3.2)\}$ is unique.

\section{Existence:}

Let us define a map $\Lambda$ from $L^{2}\left(0, T ; H^{1}(\Omega)\right) \times L^{2}\left(0, T ; \mathbf{L}^{2}(\Omega)\right.$ using the solution $(\psi, \boldsymbol{\phi})$ of $(2.1)-(2.4)$ :

$$
\Lambda(F, \mathbf{G})=\left(\left.v_{s 1} \psi\right|_{\Gamma_{\mathrm{in}}},-\mu\left[\frac{\partial}{\partial \mathbf{n}}\left(\frac{\phi}{q_{s}}\right)\right]-(\lambda+\mu)\left[\operatorname{div}\left(\frac{\phi}{q_{s}}\right)\right] \mathbf{n}\right) .
$$

From Remark 2.13 , by the continuity of the mapping $(F, \mathbf{G}) \longrightarrow(\psi, \phi)$, the operator

$$
\Lambda: L^{2}\left(0, T ; H^{1}(\Omega)\right) \times L^{2}\left(0, T ; \mathbf{L}^{2}(\Omega)\right) \longrightarrow L^{2}\left(0, T ; L^{2}\left(\Gamma_{\mathrm{in}}\right)\right) \times L^{2}\left(0, T ; \mathbf{L}^{2}(\partial \Omega)\right)
$$

is linear and continuous. So its adjoint

$$
\Lambda^{*}: L^{2}\left(0, T ; L^{2}\left(\Gamma_{\mathrm{in}}\right)\right) \times L^{2}\left(0, T ; \mathbf{L}^{2}(\partial \Omega)\right) \longrightarrow L^{2}\left(0, T ;\left[H^{1}(\Omega)\right]^{\prime}\right) \times L^{2}\left(0, T ; \mathbf{L}^{2}(\Omega)\right)
$$

is linear and continuous. Let us denote $\Lambda^{*}(w, \boldsymbol{\xi}):=(\hat{\sigma}, \hat{\mathbf{u}})$. Then

$$
\begin{aligned}
& \int_{0}^{T}\langle\hat{\sigma}, F\rangle_{\left(\left[H^{1}(\Omega)\right]^{\prime}, H^{1}(\Omega)\right)} \mathrm{d} t+\int_{0}^{T} \int_{\Omega} \mathbf{G} . \hat{\mathbf{u}} \mathrm{d} x \mathrm{~d} t \\
& =\left\langle\Lambda^{*}(w, \boldsymbol{\xi}),(F, \mathbf{G})\right\rangle_{\left[L^{2}\left(0, T ;\left[H^{1}(\Omega)\right]^{\prime}\right) \times L^{2}\left(0, T ; \mathbf{L}^{2}(\Omega)\right) ; L^{2}\left(0, T ; H^{1}(\Omega)\right) \times L^{2}\left(0, T ; \mathbf{L}^{2}(\Omega)\right)\right]} \\
& =(\Lambda(F, \mathbf{G}),(w, \boldsymbol{\xi}))_{L^{2}\left(0, T ; L^{2}\left(\Gamma_{\mathrm{in}}\right)\right) \times L^{2}\left(0, T ; \mathbf{L}^{2}(\partial \Omega)\right)} \\
& =\int_{0}^{T} \int_{\Gamma_{\mathrm{in}}} v_{s 1} \psi w \mathrm{~d} \mathbf{s} \mathrm{d} t-\int_{0}^{T} \int_{\partial \Omega}\left\{\mu\left[\frac{\partial}{\partial \mathbf{n}}\left(\frac{\phi}{q_{s}}\right)\right]+(\lambda+\mu)\left[\operatorname{div}\left(\frac{\phi}{q_{s}}\right)\right] \mathbf{n}\right\} \cdot \boldsymbol{\xi} \mathrm{d} \mathbf{s} \mathrm{d} t
\end{aligned}
$$

for every $(F, \mathbf{G})$ in $L^{2}\left(0, T ; H^{1}(\Omega)\right) \times L^{2}\left(0, T ; \mathbf{L}^{2}(\Omega)\right)$. Hence for $(w, \boldsymbol{\xi}) \in L^{2}\left(0, T ; L^{2}\left(\Gamma_{\mathrm{in}}\right)\right) \times L^{2}\left(0, T ; \mathbf{L}^{2}(\partial \Omega)\right)$, $(\hat{\sigma}, \hat{\mathbf{u}})$ is the solution of the system $\{(1.2),(1.3),(3.1),(3.2)\}$ in the sense of Definition 3.1 and

$$
\begin{aligned}
\|(\hat{\sigma}, \hat{\mathbf{u}})\|_{L^{2}\left(0, T ;\left[H^{1}(\Omega)\right]^{\prime}\right) \times L^{2}\left(0, T ; \mathbf{L}^{2}(\Omega)\right)} & =\left\|\Lambda^{*}(w, \boldsymbol{\xi})\right\|_{L^{2}\left(0, T ;\left[H^{1}(\Omega)\right]^{\prime}\right) \times L^{2}\left(0, T ; \mathbf{L}^{2}(\Omega)\right)} \\
& \leq\left\|\Lambda^{*}\right\|\|(w, \boldsymbol{\xi})\|_{L^{2}\left(0, T ; L^{2}\left(\Gamma_{\mathrm{in}}\right)\right) \times L^{2}\left(0, T ; \mathbf{L}^{2}(\partial \Omega)\right)} .
\end{aligned}
$$

Now we look for a strong solution when initial conditions are nonhomogeneous and boundary conditions are homogeneous for system (1.2)-(1.5). For that we study first the transport equation using the representation formula as in Theorem 2.6, but now with a lower order term and nonhomogeneous initial condition. 
Theorem 3.3. Let

$$
\left.\begin{array}{r}
\frac{\partial \sigma}{\partial t}(t, x)+\operatorname{div}\left(\sigma(t, x) \mathbf{v}_{s}(x)\right)=g(t, x) \text { in } \Omega_{T}, \\
\sigma(0, x)=\sigma_{0}(x) \text { in } \Omega, \quad \sigma(t, x)=0 \text { on }(0, T) \times \Gamma_{\mathrm{in} .}
\end{array}\right\}
$$

Under assumption (1.8) on $\mathbf{v}_{s}$, if $g \in L^{2}\left(0, T ; H^{1}(\Omega)\right), \sigma_{0} \in H^{1}(\Omega)$ and $\sigma_{0}(x)=0$ for $x \in \Gamma_{\mathrm{in}}$, then equation (3.3) has a unique strong solution $\sigma \in L^{\infty}\left(0, T ; H^{1}(\Omega)\right) \cap H^{1}\left(0, T ; L^{2}(\Omega)\right)$ and we have the following estimate:

$$
\max _{[0, T]}\|\sigma(t)\|_{H^{1}(\Omega)}+\left\|\frac{\partial \sigma}{\partial t}\right\|_{L^{2}\left(0, T ; L^{2}(\Omega)\right)} \leq C\left(\mathbf{v}_{s}, T, \Omega\right)\left[\left\|\sigma_{0}\right\|_{H^{1}(\Omega)}+\|g\|_{L^{2}\left(0, T ; H^{1}(\Omega)\right)}\right]
$$

for some constant $C\left(\mathbf{v}_{s}, T, \Omega\right)$.

To study the existence of a unique solution for the linearized momentum equation with inhomogeneous initial condition, we note that since $-B^{*}$ generates an analytic semigroup $S_{-B^{*}}(t)$ in $\mathbf{L}^{2}(\Omega),-B$ also generates an analytic semigroup $S_{-B}(t)$ in $\mathbf{L}^{2}(\Omega)$ with $D(-B)=\mathbf{H}^{2}(\Omega) \cap \mathbf{H}_{0}^{1}(\Omega)$. We recall the following theorem.

Theorem 3.4. Let $A$ be the infinitesimal generator of a strongly continuous analytic semigroup $\left\{S_{A}(t)\right\}_{t \geqslant 0}$, with domain $D(A)$ in a Hilbert space $Z$. Then for $f \in L^{2}(0, T ; Z)$ and $z_{0} \in D=\left\{z(0): z \in L^{2}(0, T ; D(A)) \cap\right.$ $\left.H^{1}(0, T ; Z)\right\}$ the Cauchy problem

$$
\begin{aligned}
& z^{\prime}(t)=A z(t)+f(t), t \in[0, T], \\
& z(0)=z_{0},
\end{aligned}
$$

admits a unique strong solution $z(t) \in L^{2}(0, T ; D(A)) \cap H^{1}(0, T ; Z)$ for any $T, 0<T<\infty$.

Proof of Theorem 3.4 can be found in the book "Representation and Control of Infinite Dimensional Systems" [1] (Thm. 3.1 in Sect. 3.6 of Part II, Chap. 1).

Remark 3.5. Girinon also studies the continuity equation (3.3) using semigroup theory when $g \in$ $L^{2}\left(0, T ; L^{2}(\Omega)\right), \sigma_{0} \in L^{2}(\Omega)$ in [5] (Chap. IV, Sect. 2.4) and the linearized momentum equation, which is of the form (3.5)-(3.6) (taking $A=-B, z_{0}=\mathbf{u}_{\mathbf{0}}$ ) in [5] (Chap. IV, Sect. 3) using variational method when $f \in L^{2}\left(0, T ; \mathbf{H}^{-1}(\Omega)\right)$ and $z_{0} \in \mathbf{L}^{2}(\Omega)$ and gets a weak solution. Since we have to do some integration by parts in the next theorem, we consider (3.3) and (3.5)-(3.6) with more regular initial conditions $\sigma_{0}, z_{0}$ and force terms $g, f$ in Theorems 3.3 and 3.4 and we obtain a strong solution.

Theorems 3.3 and 3.4 will be required in the following to show the well posedness of system (1.2)-(1.5).

Theorem 3.6. For every $(w, \boldsymbol{\xi}) \in L^{2}\left(0, T ; L^{2}\left(\Gamma_{\text {in }}\right)\right) \times L^{2}\left(0, T ; \mathbf{L}^{2}(\partial \Omega)\right)$ and every $\left(\sigma_{0}, \mathbf{u}_{\mathbf{0}}\right) \in L^{2}(\Omega) \times \mathbf{L}^{2}(\Omega)$, the system (1.2)-(1.5) admits a unique solution $(\sigma, \mathbf{u}) \in L^{2}\left(0, T ;\left[H^{1}(\Omega)\right]^{\prime}\right) \times L^{2}\left(0, T ; \mathbf{L}^{2}(\Omega)\right)$ in the sense of transposition as in Definition 3.1.

Proof.

(i) Uniqueness:

If $\left(\sigma_{0}, \mathbf{u}_{\mathbf{0}}\right)=(0, \mathbf{0})$ and $(w, \boldsymbol{\xi})=(0, \mathbf{0})$, we have

$$
\int_{0}^{T}\langle\sigma, F\rangle_{\left(\left[H^{1}(\Omega)\right]^{\prime}, H^{1}(\Omega)\right)} \mathrm{d} t+\int_{0}^{T} \int_{\Omega} \mathbf{G} \cdot \mathbf{u} \mathrm{d} x \mathrm{~d} t=0
$$

for all $(F, \mathbf{G}) \in L^{2}\left(0, T ; H^{1}(\Omega)\right) \times L^{2}\left(0, T ; \mathbf{L}^{2}(\Omega)\right)$. Thus $(\sigma, \mathbf{u})=(0, \mathbf{0})$ and so the solution to the linearized system $(1.2)-(1.5)$ is unique. 


\section{(ii) Existence:}

Now our target is to show that $(\sigma, \mathbf{u})=(\check{\sigma}, \check{\mathbf{u}})+(\hat{\sigma}, \hat{\mathbf{u}})$ is the solution of equation $(1.2)-(1.5)$ in the sense of Definition 3.1, where $(\check{\sigma}, \breve{\mathbf{u}})$ is the solution of the system $(3.8)-(3.11)$ corresponding to homogeneous boundary condition and nonhomogeneous initial condition. The other part $(\hat{\sigma}, \hat{\mathbf{u}})$ is the solution of the system $\{(1.2),(1.3),(3.1),(3.2)\}$ corresponding to nonhomogeneous boundary condition with homogeneous initial condition, already studied in Theorem 3.2 .

Step 1. From Theorem 3.2, we have $\Lambda^{*}(w, \boldsymbol{\xi})=(\hat{\sigma}, \hat{\mathbf{u}}) \in L^{2}\left(0, T ;\left[H^{1}(\Omega)\right]^{\prime}\right) \times L^{2}\left(0, T ; \mathbf{L}^{2}(\Omega)\right)$ and for all $(F, \mathbf{G}) \in L^{2}\left(0, T ; H^{1}(\Omega)\right) \times L^{2}\left(0, T ; \mathbf{L}^{2}(\Omega)\right)$,

$$
\begin{aligned}
& \int_{0}^{T}\langle\hat{\sigma}, F\rangle{ }_{\left(\left[H^{1}(\Omega)\right]^{\prime}, H^{1}(\Omega)\right)} \mathrm{d} t+\int_{0}^{T} \int_{\Omega} \mathbf{G} \cdot \hat{\mathbf{u}} \mathrm{d} x \mathrm{~d} t \\
= & \int_{0}^{T} \int_{\Gamma_{\mathrm{in}}} w \psi v_{s 1} \mathrm{~d} \mathbf{s} \mathrm{d} t-\int_{0}^{T} \int_{\partial \Omega}\left\{\mu\left[\frac{\partial}{\partial \mathbf{n}}\left(\frac{\phi}{q_{s}}\right)\right]+(\lambda+\mu)\left[\operatorname{div}\left(\frac{\phi}{q_{s}}\right)\right] \mathbf{n}\right\} \cdot \boldsymbol{\xi} \mathrm{d} \mathbf{s} \mathrm{d} t .
\end{aligned}
$$

Step 2. From Girinon's thesis [5] and [4], we know that for $\left(\sigma_{0}, \mathbf{u}_{\mathbf{0}}\right) \in L^{2}(\Omega) \times \mathbf{L}^{2}(\Omega)$, the following system in $\Omega_{T}$,

$$
\begin{gathered}
\frac{\partial \check{\sigma}}{\partial t}+\operatorname{div}\left(\check{\sigma} \mathbf{v}_{s}\right)=-\operatorname{div}\left(q_{s} \check{\mathbf{u}}\right), \\
\frac{\partial \check{\mathbf{u}}}{\partial t}-\frac{\mu}{q_{s}} \Delta \check{\mathbf{u}}-\frac{(\lambda+\mu)}{q_{s}} \nabla(\operatorname{div} \check{\mathbf{u}})+\left(\mathbf{v}_{s} \cdot \nabla\right) \check{\mathbf{u}}+(\check{\mathbf{u}} \cdot \nabla) \mathbf{v}_{s}+\frac{\check{\sigma}}{q_{s}}\left(\mathbf{v}_{s} \cdot \nabla\right) \mathbf{v}_{s}+a \gamma q_{s}^{\gamma-2} \nabla \check{\sigma}=\frac{\check{\sigma}}{q_{s}} \mathbf{f}, \\
\check{\sigma}(0, x)=\sigma_{0}(x), \check{\mathbf{u}}(0, x)=\mathbf{u}_{\mathbf{0}}(x) \text { in } \Omega, \\
\check{\sigma}(t, x)=0 \text { on }(0, T) \times \Gamma_{\mathrm{in}}, \quad \check{\mathbf{u}}(t, x)=\mathbf{0} \text { on } \Sigma_{T},
\end{gathered}
$$

has a unique solution $(\check{\sigma}, \check{\mathbf{u}}) \in L^{2}\left(0, T ; L^{2}(\Omega)\right) \times L^{2}\left(0, T ; \mathbf{H}_{0}^{1}(\Omega)\right)$.

In this step we will show that for $\left(\sigma_{0}, \mathbf{u}_{\mathbf{0}}\right) \in L^{2}(\Omega) \times \mathbf{L}^{2}(\Omega)$ this weak solution $(\check{\sigma}, \check{\mathbf{u}}) \in L^{2}\left(0, T ; L^{2}(\Omega)\right) \times$ $L^{2}\left(0, T ; \mathbf{H}_{0}^{1}(\Omega)\right)$ satisfies:

$$
\int_{0}^{T} \int_{\Omega} F \check{\sigma} \mathrm{d} x \mathrm{~d} t+\int_{0}^{T} \int_{\Omega} \mathbf{G} \cdot \check{\mathbf{u}} \mathrm{d} x \mathrm{~d} t=\int_{\Omega} \sigma_{0} \psi(0, x) \mathrm{d} x+\int_{\Omega} \mathbf{u}_{0} \cdot \phi(0, x) \mathrm{d} x
$$

for all $(F, \mathbf{G}) \in L^{2}\left(0, T ; H^{1}(\Omega)\right) \times L^{2}\left(0, T ; \mathbf{L}^{2}(\Omega)\right)$.

Case 1. Let us consider first the regular case when $\sigma_{0} \in H^{1}(\Omega), \sigma_{0}(x)=0 \forall x \in \Gamma_{\text {in }}$ and $\mathbf{u}_{0} \in \mathbf{H}^{2}(\Omega) \cap \mathbf{H}_{0}^{1}(\Omega)$. From Theorems 3.3 and 3.4, we get that the solution $(\tilde{\sigma}, \tilde{\mathbf{u}})$ of $(3.8)-(3.11)$ belongs to $\left[L^{\infty}\left(0, T ; H^{1}(\Omega)\right) \cap\right.$ $\left.H^{1}\left(0, T ; L^{2}(\Omega)\right)\right] \times\left[L^{2}\left(0, T ; \mathbf{H}^{2}(\Omega) \cap \mathbf{H}_{0}^{1}(\Omega)\right) \cap H^{1}\left(0, T ; \mathbf{L}^{2}(\Omega)\right)\right]$ and so the integration by parts is justified in these spaces.

Multiplying (2.1) by $\tilde{\sigma}$, using integration by parts, $\psi(T, x)=0, \mathbf{v}_{s} \cdot \mathbf{n}=0$ on $\Gamma_{0}, \tilde{\sigma}=0$ on $\Gamma_{\mathrm{in}}, \psi=0$ on $\Gamma_{\text {out }}$ and (3.8) we get

$$
\begin{aligned}
\int_{0}^{T} \int_{\Omega} \tilde{\sigma}\left[\frac{\left[\mathbf{f}-\left(\mathbf{v}_{s} \cdot \nabla\right) \mathbf{v}_{s}\right]}{q_{s}} \cdot \phi+a \gamma \operatorname{div}\left(q_{s}^{\gamma-2} \phi\right)+F\right] \mathrm{d} x \mathrm{~d} t & =-\int_{0}^{T} \int_{\Omega} \tilde{\sigma} \frac{\partial \psi}{\partial t} \mathrm{~d} x \mathrm{~d} t-\int_{0}^{T} \int_{\Omega} \tilde{\sigma}\left(\mathbf{v}_{s} \cdot \nabla \psi\right) \mathrm{d} x \mathrm{~d} t \\
& =\int_{\Omega} \int_{0}^{T}\left[\frac{\partial \tilde{\sigma}}{\partial t}+\operatorname{div}\left(\tilde{\sigma} \mathbf{v}_{s}\right)\right] \psi \mathrm{d} x \mathrm{~d} t+\int_{\Omega} \sigma_{0} \psi(0, x) \mathrm{d} x \\
& =\int_{\Omega} \int_{0}^{T}\left(q_{s} \tilde{\mathbf{u}}\right) \cdot \nabla \psi \mathrm{d} x \mathrm{~d} t+\int_{\Omega} \sigma_{0} \psi(0, x) \mathrm{d} x
\end{aligned}
$$


Multiplying (2.2) by $\tilde{\mathbf{u}}$, using integration by parts, $\boldsymbol{\phi}(T, x)=\mathbf{0}, \tilde{\mathbf{u}}(0, x)=\mathbf{u}_{\mathbf{0}}$, and $\tilde{\mathbf{u}}=\mathbf{0}, \boldsymbol{\phi}=\mathbf{0}$ on $\partial \Omega$ we get for $i=1,2$

$$
\begin{aligned}
& \int_{0}^{T} \int_{\Omega} \tilde{u}_{i}\left(q_{s} \frac{\partial \psi}{\partial x_{i}}+G_{i}\right) \mathrm{d} x \mathrm{~d} t=\int_{\Omega} \int_{0}^{T} \frac{\partial \tilde{u}_{i}}{\partial t} \phi_{i} \mathrm{~d} x \mathrm{~d} t+\int_{\Omega}\left(u_{0}\right)_{i}(x) \phi_{i}(0, x) \mathrm{d} x \\
& -\mu \int_{0}^{T} \int_{\Omega} \frac{\phi_{i}}{q_{s}} \triangle \tilde{u}_{i} \mathrm{~d} x \mathrm{~d} t+\int_{0}^{T} \int_{\Omega} \phi_{i} \operatorname{div}\left(\tilde{u}_{i} \mathbf{v}_{s}\right) \mathrm{d} x \mathrm{~d} t \\
& +(-1)^{i} \int_{0}^{T} \int_{\Omega} \tilde{u}_{i} \nabla v_{s(3-i)} \cdot\left(-\phi_{2}, \phi_{1}\right) \mathrm{d} x \mathrm{~d} t-(\lambda+\mu) \int_{0}^{T} \int_{\Omega} \tilde{u}_{i} \frac{\partial}{\partial x_{i}}\left[\operatorname{div}\left(\frac{\phi}{q_{s}}\right)\right] \mathrm{d} x \mathrm{~d} t .
\end{aligned}
$$

Thus for $i=1,2$

$$
\begin{aligned}
& \int_{0}^{T} \int_{\Omega} \tilde{u}_{i}\left(q_{s} \frac{\partial \psi}{\partial x_{i}}+G_{i}\right) \mathrm{d} x \mathrm{~d} t=\int_{\Omega} \int_{0}^{T} \frac{\partial \tilde{u}_{i}}{\partial t} \phi_{i} \mathrm{~d} x \mathrm{~d} t-\mu \int_{0}^{T} \int_{\Omega} \frac{\phi_{i}}{q_{s}} \triangle \tilde{u}_{i} \mathrm{~d} x \mathrm{~d} t \\
& +\int_{\Omega}\left(u_{0}\right)_{i}(x) \phi_{i}(0, x) \mathrm{d} x-(\lambda+\mu) \int_{0}^{T} \int_{\Omega} \tilde{u}_{i} \frac{\partial}{\partial x_{i}}\left[\operatorname{div}\left(\frac{\phi}{q_{s}}\right)\right] \mathrm{d} x \mathrm{~d} t+\int_{0}^{T} \int_{\Omega} \phi_{i}\left(\mathbf{v}_{s} \cdot \nabla \tilde{u}_{i}\right) \mathrm{d} x \mathrm{~d} t \\
& +\int_{0}^{T} \int_{\Omega} \tilde{u}_{i}\left[\phi_{1} \frac{\partial v_{s 1}}{\partial x_{i}}+\phi_{2} \frac{\partial v_{s 2}}{\partial x_{i}}\right] \mathrm{d} x \mathrm{~d} t .
\end{aligned}
$$

Using integration by parts and $\phi=0=\tilde{\mathbf{u}}$ on $\partial \Omega$, we get

$$
\int_{\Omega} \nabla\left[\operatorname{div}\left(\frac{\phi}{q_{s}}\right)\right] \cdot \tilde{\mathbf{u}} \mathrm{d} x=-\int_{\Omega} \operatorname{div}\left(\frac{\phi}{q_{s}}\right) \operatorname{div} \tilde{\mathbf{u}} \mathrm{d} x=\int_{\Omega} \frac{\phi}{q_{s}} \cdot \nabla(\operatorname{div} \tilde{\mathbf{u}}) \mathrm{d} x .
$$

Therefore, using (3.14), (3.15) and (3.9)

$$
\begin{aligned}
& \int_{0}^{T} \int_{\Omega} \tilde{\mathbf{u}} \cdot\left(q_{s} \nabla \psi+\mathbf{G}\right) \mathrm{d} x \mathrm{~d} t=\int_{\Omega} \int_{0}^{T} \frac{\partial \tilde{\mathbf{u}}}{\partial t} \cdot \boldsymbol{\phi} \mathrm{d} x \mathrm{~d} t+\int_{\Omega} \mathbf{u}_{\mathbf{0}} \cdot \boldsymbol{\phi}(0, x) \mathrm{d} x-\mu \int_{0}^{T} \int_{\Omega} \frac{\phi}{q_{s}} \cdot \Delta \tilde{\mathbf{u}} \mathrm{d} \mathrm{d} t \\
& \quad+\int_{0}^{T} \int_{\Omega}\left[(\tilde{\mathbf{u}} \cdot \nabla) \mathbf{v}_{s}+\left(\mathbf{v}_{s} \cdot \nabla\right) \tilde{\mathbf{u}}\right] \cdot \phi \mathrm{d} x \mathrm{~d} t-(\lambda+\mu) \int_{0}^{T} \int_{\Omega} \frac{\phi}{q_{s}} \cdot \nabla(\operatorname{div} \tilde{\mathbf{u}}) \mathrm{d} x \mathrm{~d} t \\
& =\int_{\Omega} \mathbf{u}_{\mathbf{0}} \cdot \phi(0, x) \mathrm{d} x+\int_{0}^{T} \int_{\Omega} \frac{\tilde{\sigma}}{q_{s}}\left[\mathbf{f}-\left(\mathbf{v}_{s} \cdot \nabla\right) \mathbf{v}_{s}\right] \cdot \phi \mathrm{d} x \mathrm{~d} t+a \gamma \int_{0}^{T} \int_{\Omega} \tilde{\sigma} \operatorname{div}\left(q_{s}^{\gamma-2} \phi\right) \mathrm{d} x \mathrm{~d} t .
\end{aligned}
$$

Thus adding (3.13) and (3.16) we get

$$
\int_{0}^{T} \int_{\Omega} F \tilde{\sigma} \mathrm{d} x \mathrm{~d} t+\int_{0}^{T} \int_{\Omega} \mathbf{G} \cdot \tilde{\mathbf{u}} \mathrm{d} x \mathrm{~d} t=\int_{\Omega} \sigma_{0} \psi(0, x) \mathrm{d} x+\int_{\Omega} \mathbf{u}_{0} \cdot \phi(0, x) \mathrm{d} x .
$$

Case 2. Let us consider the general case when $\sigma_{0} \in L^{2}(\Omega)$ and $\mathbf{u}_{\mathbf{0}} \in \mathbf{L}^{2}(\Omega)$. We will deduce equation (3.17) through a limiting procedure in this case. $C_{c}^{\infty}(\Omega)$ is dense in $L^{2}(\Omega)$. Because of this, $\left\{h_{0} \in H^{1}(\Omega): h_{0}(x)=\right.$ $\left.0 \forall x \in \Gamma_{\text {in }}\right\}$ is dense in $L^{2}(\Omega)$, so is $\mathbf{H}^{2}(\Omega) \cap \mathbf{H}_{0}^{1}(\Omega)$ in $\mathbf{L}^{2}(\Omega)$. So there exist sequences $\left(\sigma_{0}\right)_{n} \in\left\{h_{0} \in H^{1}(\Omega)\right.$ : $\left.h_{0}(x)=0 \forall x \in \Gamma_{\text {in }}\right\}$ and $\left(\mathbf{u}_{\mathbf{0}}\right)_{n} \in \mathbf{H}^{2}(\Omega) \cap \mathbf{H}_{0}^{1}(\Omega)$ such that

$$
\left(\sigma_{0}\right)_{n} \longrightarrow \sigma_{0} \text { in } L^{2}(\Omega) \text { and }\left(\mathbf{u}_{\mathbf{0}}\right)_{n} \longrightarrow \mathbf{u}_{\mathbf{0}} \text { in } \mathbf{L}^{2}(\Omega) .
$$

Let $\left(\tilde{\sigma}_{n}, \tilde{\mathbf{u}}_{n}\right) \in\left[L^{\infty}\left(0, T ; H^{1}(\Omega)\right) \cap H^{1}\left(0, T ; L^{2}(\Omega)\right)\right] \times\left[L^{2}\left(0, T ; \mathbf{H}^{2}(\Omega) \cap \mathbf{H}_{0}^{1}(\Omega)\right) \cap H^{1}\left(0, T ; \mathbf{L}^{2}(\Omega)\right)\right]$ be the solution of $(3.8)-(3.11)$ corresponding to $\left(\sigma_{0}\right)_{n}$ and $\left(\mathbf{u}_{\mathbf{0}}\right)_{n}$. Therefore by case 1 we have

$$
\int_{0}^{T} \int_{\Omega} F \tilde{\sigma}_{n} \mathrm{~d} x \mathrm{~d} t+\int_{0}^{T} \int_{\Omega} \mathbf{G} \cdot \tilde{\mathbf{u}}_{n} \mathrm{~d} x \mathrm{~d} t=\int_{\Omega}\left(\sigma_{0}\right)_{n} \psi(0, x) \mathrm{d} x+\int_{\Omega}\left(\mathbf{u}_{\mathbf{0}}\right)_{n} \cdot \boldsymbol{\phi}(0, x) \mathrm{d} x .
$$


Since the solution map corresponding to homogeneous system (3.8)-(3.11) is linear and continuous,

$$
\left\|\tilde{\sigma}_{n}\right\|_{L^{2}\left(0, T ; L^{2}(\Omega)\right)}+\left\|\tilde{\mathbf{u}}_{n}\right\|_{L^{2}\left(0, T ; \mathbf{H}_{0}^{1}(\Omega)\right)} \leq C\left(\mathbf{v}_{s}, T\right)\left[\left\|\left(\sigma_{0}\right)_{n}\right\|_{L^{2}(\Omega)}+\left\|\left(\mathbf{u}_{\mathbf{0}}\right)_{n}\right\|_{\mathbf{L}^{2}(\Omega)}\right] .
$$

Thus we get $\left(\tilde{\sigma}_{n}, \tilde{\mathbf{u}}_{n}\right)$ is a Cauchy sequence in $L^{2}\left(0, T ; L^{2}(\Omega)\right) \times L^{2}\left(0, T ; \mathbf{H}_{0}^{1}(\Omega)\right)$. Let $\left(\tilde{\sigma}_{n}, \tilde{\mathbf{u}}_{n}\right)$ converge to $(\check{\sigma}, \check{\mathbf{u}})$ in $L^{2}\left(0, T ; L^{2}(\Omega)\right) \times L^{2}\left(0, T ; \mathbf{H}_{0}^{1}(\Omega)\right)$. Hence taking the limit as $n \longrightarrow \infty$ in (3.18) and (3.19) we get

$$
\int_{0}^{T} \int_{\Omega} F \check{\sigma} \mathrm{d} x \mathrm{~d} t+\int_{0}^{T} \int_{\Omega} \mathbf{G} \cdot \check{\mathbf{u}} \mathrm{d} x \mathrm{~d} t=\int_{\Omega} \sigma_{0} \psi(0, x) \mathrm{d} x+\int_{\Omega} \mathbf{u}_{0} \cdot \boldsymbol{\phi}(0, x) \mathrm{d} x
$$

and

$$
\|\check{\sigma}\|_{L^{2}\left(0, T ; L^{2}(\Omega)\right)}+\|\check{\mathbf{u}}\|_{L^{2}\left(0, T ; \mathbf{H}_{0}^{1}(\Omega)\right)} \leq C\left(\mathbf{v}_{s}, T\right)\left[\left\|\sigma_{0}\right\|_{L^{2}(\Omega)}+\left\|\mathbf{u}_{\mathbf{0}}\right\|_{\mathbf{L}^{2}(\Omega)}\right]
$$

for all $(F, \mathbf{G}) \in L^{2}\left(0, T ; H^{1}(\Omega)\right) \times L^{2}\left(0, T ; \mathbf{L}^{2}(\Omega)\right)$ and $\left(\sigma_{0}, \mathbf{u}_{0}\right) \in L^{2}(\Omega) \times \mathbf{L}^{2}(\Omega)$.

Hence adding (3.7) and (3.12) we get $(\sigma, \mathbf{u})=(\check{\sigma}, \check{\mathbf{u}})+(\hat{\sigma}, \hat{\mathbf{u}})$ is the solution of equations (1.2)-(1.5) in the sense of Definition 3.1.

Theorem 3.7. Let $H$ be defined on $L^{2}(\Omega) \times \mathbf{L}^{2}(\Omega) \times L^{2}\left(0, T ; L^{2}\left(\Gamma_{\text {in }}\right)\right) \times L^{2}\left(0, T ; \mathbf{L}^{2}(\partial \Omega)\right)$ by

$$
H\left(\sigma_{0}, \mathbf{u}_{\mathbf{0}}, w, \boldsymbol{\xi}\right)=(\sigma, \mathbf{u}) .
$$

Then $H$ is linear and continuous from $L^{2}(\Omega) \times \mathbf{L}^{2}(\Omega) \times L^{2}\left(0, T ; L^{2}\left(\Gamma_{\mathrm{in}}\right)\right) \times L^{2}\left(0, T ; \mathbf{L}^{2}(\partial \Omega)\right)$ into $L^{2}\left(0, T ;\left[H^{1}(\Omega)\right]^{\prime}\right) \times L^{2}\left(0, T ; \mathbf{L}^{2}(\Omega)\right)$ and there exists a constant $C$ such that

$$
\|\sigma\|_{L^{2}\left(0, T ;\left[H^{1}(\Omega)\right]^{\prime}\right)}+\|\mathbf{u}\|_{L^{2}\left(0, T ; \mathbf{L}^{2}(\Omega)\right)} \leq C\left[\left\|\sigma_{0}\right\|_{L^{2}(\Omega)}+\left\|\mathbf{u}_{\mathbf{0}}\right\|_{\mathbf{L}^{2}(\Omega)}+\|w\|_{L^{2}\left(0, T ; L^{2}\left(\Gamma_{\mathrm{in}}\right)\right)}+\|\boldsymbol{\xi}\| \|_{L^{2}\left(0, T ; \mathbf{L}^{2}(\partial \Omega)\right)}\right] .
$$

Proof. Clearly $\mathrm{H}$ is linear. To verify the continuity of $H$,

$$
\begin{aligned}
& \left\|H\left(\sigma_{0}, \mathbf{u}_{\mathbf{0}}, w, \boldsymbol{\xi}\right)\right\|_{L^{2}\left(0, T ;\left[H^{1}(\Omega)\right]^{\prime}\right) \times L^{2}\left(0, T ; \mathbf{L}^{2}(\Omega)\right)}=\left\|\Lambda^{*}(w, \boldsymbol{\xi})+(\check{\sigma}, \check{\mathbf{u}})\right\|_{L^{2}\left(0, T ;\left[H^{1}(\Omega)\right]^{\prime}\right) \times L^{2}\left(0, T ; \mathbf{L}^{2}(\Omega)\right)} \\
& \leq\left\|\Lambda^{*}\right\|\|(w, \boldsymbol{\xi})\|_{L^{2}\left(0, T ; L^{2}\left(\Gamma_{\mathrm{in}}\right)\right) \times L^{2}\left(0, T ; \mathbf{L}^{2}(\partial \Omega)\right)}+\|\check{\sigma}\|_{L^{2}\left(0, T ; L^{2}(\Omega)\right)}+\|\check{\mathbf{u}}\|_{L^{2}\left(0, T ; \mathbf{L}^{2}(\Omega)\right)} \\
& \leq C\left[\|w\|_{L^{2}\left(0, T ; L^{2}\left(\Gamma_{\mathrm{in}}\right)\right)}+\|\boldsymbol{\xi}\|_{L^{2}\left(0, T ; \mathbf{L}^{2}(\partial \Omega)\right)}+\left\|\sigma_{0}\right\|_{L^{2}(\Omega)}+\left\|\mathbf{u}_{\mathbf{0}}\right\|_{\mathbf{L}^{2}(\Omega)}\right]
\end{aligned}
$$

using (3.20).

Remark 3.8. We consider the system (1.2)-(1.5) with less regular boundary data, namely $L^{2}$ boundary data, so that we get the solution via transposition in weaker spaces. If boundary data are little bit regular, then solution of (1.2)-(1.5) has better regularity. In fact we show in the following that, if boundary data only for the velocity has better space and time regularity, then solution of (1.2)-(1.5) will be more regular.

Let $w \in L^{2}\left(0, T ; L^{2}\left(\Gamma_{\text {in }}\right)\right)$ and $\boldsymbol{\xi} \in H^{1}\left(0, T ; \mathbf{H}^{\frac{1}{2}}(\partial \Omega)\right)$, then using the surjectivity of trace map $T$ from $\mathbf{H}^{1}(\Omega)$ on to $\mathbf{H}^{\frac{1}{2}}(\partial \Omega)$ for our domain, a reactangle with Lipschitz boundary, we can pick a $\tilde{\boldsymbol{\xi}} \in H^{1}\left(0, T ; \mathbf{H}^{1}(\Omega)\right)$ such that $T(\tilde{\boldsymbol{\xi}})=\boldsymbol{\xi}$. Thus by this lifting arument we obtain a homogeneous boundary value problem for $(\mathbf{u}-\tilde{\boldsymbol{\xi}})$ and hence using [5] (Sect. 3 of Chap. 4 in Girinon) we get $\mathbf{u}$ in $L^{2}\left(0, T ; \mathbf{H}^{1}(\Omega)\right) \cap C\left([0, T] ; \mathbf{L}^{2}(\Omega)\right)$ ). For the density we first consider adjoint continuity equation for $\psi$ with force term $\varphi$ in $L^{2}\left(0, T ; L^{2}(\Omega)\right)$ and using multiplier method (namely multiplying by $\psi$ ), we show the mild solution $\psi \in C\left([0, T] ; L^{2}(\Omega)\right.$ ) has a trace on inflow boundary (hidden regularity), in fact $\left.\psi\right|_{\Gamma_{\mathrm{in}}} \in L^{2}\left(0, T ; L^{2}\left(\Gamma_{\mathrm{in}}\right)\right)$ and the map $\left.\varphi \rightarrow \psi\right|_{\Gamma_{\mathrm{in}}}$ is continuous from $L^{2}\left(0, T ; L^{2}(\Omega)\right)$ into $L^{2}\left(0, T ; L^{2}\left(\Gamma_{\text {in }}\right)\right)$ (For details see Sect. 5.2 in appendix). Then using transposition method for the continuity equation we will get $\sigma \in L^{2}\left(0, T ; L^{2}(\Omega)\right)$ for $w \in L^{2}\left(0, T ; L^{2}\left(\Gamma_{\text {in }}\right)\right)$. After that defining a contraction map in $L^{2}\left(0, T_{1}, L^{2}(\Omega)\right)$ exactly like Girinon [5] (Sect. 4 of Chap. 4) for this nonhomogeneous boundary conditions, we get the solution $(\sigma, \mathbf{u}) \in L^{2}\left(0, T_{1} ; L^{2}(\Omega)\right) \times\left[L^{2}\left(0, T_{1} ; \mathbf{H}^{1}(\Omega)\right) \cap C\left(\left[0, T_{1}\right] ; \mathbf{L}^{2}(\Omega)\right)\right]$ and then in $L^{2}\left(0, T ; L^{2}(\Omega)\right) \times\left[L^{2}\left(0, T ; \mathbf{H}^{1}(\Omega)\right) \cap C\left([0, T] ; \mathbf{L}^{2}(\Omega)\right)\right]$. 


\section{Optimal Control problem}

In this section we study the optimal control problem $(\mathrm{P})$ mentioned in the introduction of this paper. First we discuss the norm $\|\mid\|_{\left[H^{1}(\Omega)\right]^{\prime}}$ and then prove the existence of a unique solution to the problem (P) and derive the optimality system.

Denote by $\|\cdot\|_{\left[H^{1}(\Omega)\right]^{\prime}}$ the dual norm,

$$
\|f\|_{\left[H^{1}(\Omega)\right]^{\prime}}=\sup _{z \in H^{1}(\Omega)} \frac{\langle f, z\rangle_{\left(\left[H^{1}(\Omega)\right]^{\prime}, H^{1}(\Omega)\right)}}{\|z\|_{H^{1}(\Omega)}} .
$$

For $f$ in $\left[H^{1}(\Omega)\right]^{\prime}$, let $u$ be the solution to the equation

$$
-\Delta u+u=f \text { in } \Omega, \quad \frac{\partial u}{\partial n}=0 \text { on } \partial \Omega
$$

Denote $u=(-\Delta+I)^{-1} f$.

Proposition 4.1. If we define

$$
\|\| f \|_{\left[H^{1}(\Omega)\right]^{\prime}}:=\left\langle f,(-\Delta+I)^{-1} f\right\rangle_{\left(\left[H^{1}(\Omega)\right]^{\prime}, H^{1}(\Omega)\right)}^{\frac{1}{2}},
$$

then it is a norm on $\left[H^{1}(\Omega)\right]^{\prime}$ equivalent to the usual norm $\|f\|_{\left[H^{1}(\Omega)\right]^{\prime}}$.

It is well known, so we omit the proof here.

\subsection{Existence and uniqueness of solution to $(\mathrm{P})$}

Theorem 4.2. Under assumptions (1.6)-(1.8), the control problem $(P)$ admits a unique solution in $L^{2}\left(0, T ; L^{2}\left(\Gamma_{\mathrm{in}}\right)\right) \times L^{2}\left(0, T ; \mathbf{L}^{2}(\partial \Omega)\right)$.

Proof.

(i) Existence:

Let

$$
\bar{m}=\inf _{(w, \boldsymbol{\xi}) \in L^{2}\left(0, T ; L^{2}\left(\Gamma_{\mathrm{in}}\right)\right) \times L^{2}\left(0, T ; \mathbf{L}^{2}(\partial \Omega)\right)} J(\sigma, \mathbf{u}, w, \boldsymbol{\xi}) .
$$

So there exists a minimizing sequence $\left(w_{n}, \boldsymbol{\xi}_{n}\right) \in L^{2}\left(0, T ; L^{2}\left(\Gamma_{\mathrm{in}}\right)\right) \times L^{2}\left(0, T ; \mathbf{L}^{2}(\partial \Omega)\right)$ such that

$$
\lim _{n \rightarrow \infty} J\left(\sigma_{n}, \mathbf{u}_{n}, w_{n}, \boldsymbol{\xi}_{n}\right)=\bar{m},
$$

where $\left(\sigma_{n}, \mathbf{u}_{n}\right)$ is the solution of system (1.2)-(1.5) corresponding to the boundary value $w_{n}, \boldsymbol{\xi}_{n}$.

Now

$$
\lim _{n \rightarrow \infty} J\left(\sigma_{n}, \mathbf{u}_{n}, w_{n}, \boldsymbol{\xi}_{n}\right)=\bar{m} \Rightarrow J\left(\sigma_{n}, \mathbf{u}_{n}, w_{n}, \boldsymbol{\xi}_{n}\right) \leq D, \text { for some constant } D .
$$

Thus $w_{n}, \boldsymbol{\xi}_{n}, \sigma_{n}, \mathbf{u}_{n}$ are bounded sequence in $L^{2}\left(0, T ; L^{2}\left(\Gamma_{\text {in }}\right)\right), L^{2}\left(0, T ; \mathbf{L}^{2}(\partial \Omega)\right), L^{2}\left(0, T ;\left[H^{1}(\Omega)\right]^{\prime}\right)$ and $L^{2}\left(0, T ; \mathbf{L}^{2}(\Omega)\right)$. So there exist subsequences of $w_{n}, \boldsymbol{\xi}_{n}, \sigma_{n}, \mathbf{u}_{n}$ (still indexed by $\mathrm{n}$ to simplify the notation) and functions $w, \boldsymbol{\xi}, \sigma, \mathbf{u}$ such that

$$
\begin{aligned}
& w_{n} \rightarrow w \text { in } L^{2}\left(0, T ; L^{2}\left(\Gamma_{\mathrm{in}}\right)\right), \\
& \boldsymbol{\xi}_{n} \rightarrow \boldsymbol{\xi} \text { in } L^{2}\left(0, T ; \mathbf{L}^{2}(\partial \Omega)\right), \\
& \sigma_{n} \rightarrow \sigma \text { in } L^{2}\left(0, T ;\left[H^{1}(\Omega)\right]^{\prime}\right), \\
& \mathbf{u}_{n} \rightarrow \mathbf{u} \text { in } L^{2}\left(0, T ; \mathbf{L}^{2}(\Omega)\right) .
\end{aligned}
$$


So

$$
\begin{aligned}
\|w\|_{L^{2}\left(0, T ; L^{2}\left(\Gamma_{\mathrm{in}}\right)\right)}^{2} & \leq \liminf _{n \rightarrow \infty}\left\|w_{n}\right\|_{L^{2}\left(0, T ; L^{2}\left(\Gamma_{\mathrm{in}}\right)\right)}^{2}, \\
\|\boldsymbol{\xi}\|_{L^{2}\left(0, T ; \mathbf{L}^{2}(\partial \Omega)\right)}^{2} & \leq \liminf _{n \rightarrow \infty}\left\|\boldsymbol{\xi}_{n}\right\|_{L^{2}\left(0, T ; \mathbf{L}^{2}(\partial \Omega)\right)}^{2}, \\
\left\|\sigma-\sigma^{d}\right\|_{L^{2}\left(0, T ;\left[H^{1}(\Omega)\right]^{\prime}\right)}^{2} & \leq \liminf _{n \rightarrow \infty}\left\|\sigma_{n}-\sigma^{d}\right\|_{L^{2}\left(0, T ;\left[H^{1}(\Omega)\right]^{\prime}\right)}^{2}, \\
\left\|\mathbf{u}-\mathbf{u}^{d}\right\|_{L^{2}\left(0, T ; \mathbf{L}^{2}(\Omega)\right)}^{2} & \leq \liminf _{n \rightarrow \infty}\left\|\mathbf{u}_{n}-\mathbf{u}^{d}\right\|_{L^{2}\left(0, T ; \mathbf{L}^{2}(\Omega)\right)}^{2}
\end{aligned}
$$

Therefore from (4.6)-(4.9) we get

$$
J(\sigma, \mathbf{u}, w, \boldsymbol{\xi}) \leq \liminf _{n \rightarrow \infty} J\left(\sigma_{n}, \mathbf{u}_{n}, w_{n}, \boldsymbol{\xi}_{n}\right)=\bar{m} .
$$

Hence $J(\sigma, \mathbf{u}, w, \boldsymbol{\xi})=\bar{m}$. Now the proof of existence of optimal solution will be complete if we can show that $(\sigma, \mathbf{u})$ is the solution of system (1.2)-(1.5) corresponding to the boundary value $(w, \boldsymbol{\xi})$. As $\left(\sigma_{n}, \mathbf{u}_{n}\right)$ is the solution of system (1.2)-(1.5) corresponding to the boundary value $w_{n}, \boldsymbol{\xi}_{n}$, we have using Definition 3.1 of transposition:

$$
\begin{array}{r}
\int_{0}^{T}\left\langle\sigma_{n}, F\right\rangle_{\left(\left[H^{1}(\Omega)\right]^{\prime}, H^{1}(\Omega)\right)} \mathrm{d} t+\int_{0}^{T} \int_{\Omega} \mathbf{G} \cdot \mathbf{u}_{n} \mathrm{~d} x \mathrm{~d} t=\int_{\Omega} \sigma_{0} \psi(0, x) \mathrm{d} x+\int_{\Omega} \mathbf{u}_{\mathbf{0}} \cdot \boldsymbol{\phi}(0, x) \mathrm{d} x \\
\quad+\int_{0}^{T} \int_{\Gamma_{\mathrm{in}}} w_{n} \psi v_{s 1} \mathrm{~d} \mathbf{s} \mathrm{d} t-\int_{0}^{T} \int_{\partial \Omega}\left\{\mu\left[\frac{\partial}{\partial \mathbf{n}}\left(\frac{\phi}{q_{s}}\right)\right]+(\lambda+\mu)\left[\operatorname{div}\left(\frac{\phi}{q_{s}}\right)\right] \mathbf{n}\right\} \cdot \boldsymbol{\xi}_{n} \mathrm{~d} \mathbf{s} \mathrm{d} t
\end{array}
$$

for all $(F, \mathbf{G}) \in L^{2}\left(0, T ; H^{1}(\Omega)\right) \times L^{2}\left(0, T ; \mathbf{L}^{2}(\Omega)\right)$, where $(\psi, \phi)$ is a solution to adjoint system (2.1)-(2.4).

Now using (4.2)-(4.5) and taking limit in the above equation we get

$$
\begin{array}{r}
\int_{0}^{T}\langle\sigma, F\rangle_{\left(\left[H^{1}(\Omega)\right]^{\prime}, H^{1}(\Omega)\right)} \mathrm{d} t+\int_{0}^{T} \int_{\Omega} \mathbf{G} \cdot \mathbf{u} \mathrm{d} x \mathrm{~d} t=\int_{\Omega} \sigma_{0} \psi(0, x) \mathrm{d} x+\int_{\Omega} \mathbf{u}_{\mathbf{0}} \cdot \boldsymbol{\phi}(0, x) \mathrm{d} x \\
+\int_{0}^{T} \int_{\Gamma_{\mathrm{in}}} w \psi v_{s 1} \mathrm{~d} \mathbf{s} \mathrm{d} t-\int_{0}^{T} \int_{\partial \Omega}\left\{\mu\left[\frac{\partial}{\partial \mathbf{n}}\left(\frac{\phi}{q_{s}}\right)\right]+(\lambda+\mu)\left[\operatorname{div}\left(\frac{\phi}{q_{s}}\right)\right] \mathbf{n}\right\} \cdot \boldsymbol{\xi} \mathrm{d} \mathbf{s} \mathrm{d} t
\end{array}
$$

for all $(F, \mathbf{G}) \in L^{2}\left(0, T ; H^{1}(\Omega)\right) \times L^{2}\left(0, T ; \mathbf{L}^{2}(\Omega)\right)$, where $(\psi, \phi)$ is a solution to the adjoint system $(2.1)-(2.4)$ and hence $(\sigma, \mathbf{u})$ is the solution of the system $(1.2)-(1.5)$.

(ii) Uniqueness:

Since $\mathrm{J}$ is strictly convex, the minimum is unique. Thus the problem $(\mathrm{P})$ admits a unique solution. This completes the proof.

\subsection{Green's formula}

To obtain the expression for the gradient of $J$, we need the following Green's formula which is a simple consequence of Definition 3.1.

Theorem 4.3. If $w \in L^{2}\left(0, T ; L^{2}\left(\Gamma_{\mathrm{in}}\right)\right), \boldsymbol{\xi} \in L^{2}\left(0, T ; \mathbf{L}^{2}(\partial \Omega)\right), F \in L^{2}\left(0, T ; H^{1}(\Omega)\right)$ and $\mathbf{G} \in L^{2}\left(0, T ; \mathbf{L}^{2}(\Omega)\right)$, then the solution $(\sigma, \mathbf{u})$ of system $\{(1.2),(1.3),(3.1),(3.2)\}$ and the solution $(\psi, \phi)$ of adjoint system $(2.1)-(2.4)$ satisfy the following

$$
\begin{aligned}
\int_{0}^{T}\langle\sigma, F\rangle_{\left(\left[H^{1}(\Omega)\right]^{\prime}, H^{1}(\Omega)\right)} \mathrm{d} t+\int_{0}^{T} \int_{\Omega} \mathbf{G} \cdot \mathbf{u} \mathrm{d} x & \mathrm{~d} t=\int_{0}^{T} \int_{\Gamma_{\mathrm{in}}} w \psi v_{s 1} \mathrm{~d} \mathbf{s} \mathrm{d} t \\
& -\int_{0}^{T} \int_{\partial \Omega}\left\{\mu\left[\frac{\partial}{\partial \mathbf{n}}\left(\frac{\phi}{q_{s}}\right)\right]+(\lambda+\mu)\left[\operatorname{div}\left(\frac{\phi}{q_{s}}\right)\right] \mathbf{n}\right\} \cdot \boldsymbol{\xi} \mathrm{d} \mathbf{s} \mathrm{d} t .
\end{aligned}
$$

This will be required to write the optimality system for problem $(\mathrm{P})$ in the next section. 


\subsection{Optimality system for $(\mathrm{P})$}

Necessary and sufficient optimality conditions are stated in the following theorem.

Theorem 4.4. Under assumptions (1.6)-(1.8), if $(\bar{w}, \overline{\boldsymbol{\xi}})$ is the optimal control for the problem $(P)$ and $(\bar{\sigma}, \overline{\mathbf{u}})$ is the corresponding solution of system (1.2)-(1.5), then

$$
\begin{aligned}
& \bar{w}=-\left.\frac{1}{\beta}\left\{v_{s 1} \psi\right\}\right|_{(0, T) \times \Gamma_{\mathrm{in}}}, \\
& \overline{\boldsymbol{\xi}}=-\frac{1}{\beta}\left\{\mu\left[\frac{\partial}{\partial \mathbf{n}}\left(\frac{\phi}{q_{s}}\right)\right]+(\lambda+\mu)\left[\operatorname{div}\left(\frac{\phi}{q_{s}}\right)\right] \mathbf{n}\right\},
\end{aligned}
$$

where $(\psi, \phi)$ is the solution of the adjoint system (2.1)-(2.4) corresponding to

$$
F=(-\Delta+I)^{-1}\left(\bar{\sigma}-\sigma^{d}\right), \quad \mathbf{G}=\overline{\mathbf{u}}-\mathbf{u}^{d} .
$$

Conversely, if a pair $((\tilde{\sigma}, \tilde{\mathbf{u}}),(\tilde{\psi}, \tilde{\boldsymbol{\phi}}))$ obeys the coupled system

$$
\begin{gathered}
\frac{\partial \sigma}{\partial t}+\operatorname{div}\left(\sigma \mathbf{v}_{s}\right)=-\operatorname{div}\left(q_{s} \mathbf{u}\right) \quad \text { in } \Omega_{T}, \\
\frac{\partial \mathbf{u}}{\partial t}-\frac{\mu}{q_{s}} \Delta \mathbf{u}-\frac{(\lambda+\mu)}{q_{s}} \nabla(\operatorname{div} \mathbf{u})+\left(\mathbf{v}_{s} \cdot \nabla\right) \mathbf{u}+(\mathbf{u} \cdot \nabla) \mathbf{v}_{s}=-a \gamma q_{s}^{\gamma-2} \nabla \sigma+\frac{\sigma}{q_{s}}\left[\mathbf{f}-\left(\mathbf{v}_{s} \cdot \nabla\right) \mathbf{v}_{s}\right] \quad \text { in } \Omega_{T}, \\
\sigma(0, x)=\sigma_{0}(x), \mathbf{u}(0, x)=\mathbf{u}_{\mathbf{0}}(x) \quad \text { in } \Omega, \\
\sigma(t, x)=-\left.\frac{1}{\beta}\left\{v_{s 1} \psi\right\}\right|_{(0, T) \times \Gamma_{\mathrm{in}}} \text { on }(0, T) \times \Gamma_{\mathrm{in}}, \\
\mathbf{u}(t, x)=-\frac{1}{\beta}\left\{\mu\left[\frac{\partial}{\partial \mathbf{n}}\left(\frac{\phi}{q_{s}}\right)\right]+(\lambda+\mu)\left[\operatorname{div}\left(\frac{\phi}{q_{s}}\right)\right] \mathbf{n}\right\} \text { on } \Sigma_{T}, \\
-\frac{\partial \phi}{\partial t}-\mathbf{v}_{s} \cdot \nabla \psi=\frac{\left[\mathbf{f}-\left(\mathbf{v}_{s} \cdot \nabla\right) \mathbf{v}_{s}\right]}{q_{s}} \cdot \boldsymbol{\phi}+a \gamma \operatorname{div}\left(q_{s}^{\gamma-2} \boldsymbol{\phi}\right)+(-\Delta+I)^{-1}\left(\sigma-\sigma^{d}\right) \text { in } \Omega_{T}, \\
\Delta\left(\frac{\phi}{q_{s}}\right)-(\lambda+\mu) \nabla\left[\operatorname{div}\left(\frac{\phi}{q_{s}}\right)\right]-\left(\operatorname{div}\left(\phi_{1} \mathbf{v}_{s}\right), \operatorname{div}\left(\phi_{2} \mathbf{v}_{s}\right)\right)+\left(\nabla \mathbf{v}_{s}\right)^{T} \boldsymbol{\phi}=q_{s} \nabla \psi+\left(\mathbf{u}-\mathbf{u}^{d}\right), \\
\psi(T, x)=0, \phi(T, x)=\mathbf{0} \text { in } \Omega, \\
\phi(t, x)=0 \text { on }(0, T) \times \Gamma_{\text {out }}, \quad \phi(t, x)=\mathbf{0} \text { on } \Sigma_{T},
\end{gathered}
$$

then the pair

$$
\left([\tilde{\sigma}, \tilde{\mathbf{u}}] ;\left[-\left.\frac{1}{\beta}\left\{v_{s 1} \tilde{\psi}\right\}\right|_{(0, T) \times \Gamma_{\mathrm{in}}},-\frac{1}{\beta}\left\{\mu\left[\frac{\partial}{\partial \mathbf{n}}\left(\frac{\tilde{\phi}}{q_{s}}\right)\right]+(\lambda+\mu)\left[\operatorname{div}\left(\frac{\tilde{\phi}}{q_{s}}\right)\right] \mathbf{n}\right\}\right]\right)
$$

is the optimal solution to problem $(P)$. 
Proof. First we obtain the necessary optimality conditions. Let

$$
\begin{aligned}
F_{1}(w, \boldsymbol{\xi})=J(\sigma, \mathbf{u}, w, \boldsymbol{\xi})= & \frac{1}{2} \int_{0}^{T}\left|\left\|\sigma-\sigma^{d}\left|\|_{\left[H^{1}(\Omega)\right]^{\prime}}^{2} \mathrm{~d} t+\frac{1}{2} \int_{0}^{T} \int_{\Omega}\right| \mathbf{u}-\left.\mathbf{u}^{d}\right|^{2} \mathrm{~d} x \mathrm{~d} t\right.\right. \\
& +\frac{\beta}{2}\left[\int_{0}^{T} \int_{\Gamma_{\mathrm{in}}} w^{2} \mathrm{~d} \mathbf{s} \mathrm{d} t+\int_{0}^{T} \int_{\partial \Omega}|\boldsymbol{\xi}|^{2} \mathrm{~d} \mathbf{s} \mathrm{d} t\right],
\end{aligned}
$$

where $H\left(\sigma_{0}, \mathbf{u}_{0}, w, \boldsymbol{\xi}\right)=(\sigma, \mathbf{u}), \mathrm{H}$ is the solution map as defined in Theorem 3.7. Our aim is to compute the gradient of $F_{1}$.

Let $(\bar{w}, \overline{\boldsymbol{\xi}})$ be the optimal control and $(\bar{\sigma}, \overline{\mathbf{u}})$ be the optimal state i.e. $H\left(\sigma_{0}, \mathbf{u}_{\mathbf{0}}, \bar{w}, \overline{\boldsymbol{\xi}}\right)=(\bar{\sigma}, \overline{\mathbf{u}})$. Let $H\left(\sigma_{0}, \mathbf{u}_{\mathbf{0}}, \bar{w}+\theta w, \overline{\boldsymbol{\xi}}+\theta \boldsymbol{\xi}\right)=\left(\sigma^{\theta}, \mathbf{u}^{\theta}\right)$. As $\mathrm{H}$ is linear, we have

$$
H(0,0, \theta w, \theta \boldsymbol{\xi})=\left(\sigma^{\theta}-\bar{\sigma}, \mathbf{u}^{\theta}-\overline{\mathbf{u}}\right) ; H(0,0, w, \boldsymbol{\xi})=\left(\frac{\sigma^{\theta}-\bar{\sigma}}{\theta}, \frac{\mathbf{u}^{\theta}-\overline{\mathbf{u}}}{\theta}\right) .
$$

Now using (3.21) of Theorem 3.7 we have

$$
\left\|\sigma^{\theta}-\bar{\sigma}\right\|_{L^{2}\left(0, T ;\left[H^{1}(\Omega)\right]^{\prime}\right)}+\left\|\mathbf{u}^{\theta}-\overline{\mathbf{u}}\right\|_{L^{2}\left(0, T ; \mathbf{L}^{2}(\Omega)\right)} \leq C|\theta|\left[\|w\|_{L^{2}\left(0, T ; L^{2}\left(\Gamma_{\mathrm{in}}\right)\right)}+\|\boldsymbol{\xi}\|_{L^{2}\left(0, T ; \mathbf{L}^{2}(\Omega)\right)}\right] .
$$

Thus

$$
\left(\sigma^{\theta}, \mathbf{u}^{\theta}\right) \longrightarrow(\bar{\sigma}, \overline{\mathbf{u}}) \text { in } L^{2}\left(0, T ;\left[H^{1}(\Omega)\right]^{\prime}\right) \times L^{2}\left(0, T ; \mathbf{L}^{2}(\Omega)\right),
$$

when $\theta \longrightarrow 0$. Let us define

$$
\left(\sigma^{w, \xi}, \mathbf{u}^{w, \xi}\right)=\left(\frac{\sigma^{\theta}-\bar{\sigma}}{\theta}, \frac{\mathbf{u}^{\theta}-\overline{\mathbf{u}}}{\theta}\right)
$$

Then $H(0,0, w, \boldsymbol{\xi})=\left(\sigma^{w, \xi}, \mathbf{u}^{w, \xi}\right)$.

$$
\begin{aligned}
& F_{1}(\bar{w}+\theta w, \overline{\boldsymbol{\xi}}+\theta \boldsymbol{\xi})-F_{1}(\bar{w}, \overline{\boldsymbol{\xi}})=\frac{1}{2} \int_{0}^{T}\left[\left\langle\sigma^{\theta}-\sigma^{d},(-\Delta+I)^{-1}\left(\sigma^{\theta}-\sigma^{d}\right)\right\rangle_{\left(\left[H^{1}(\Omega)\right]^{\prime}, H^{1}(\Omega)\right)}\right. \\
& \left.\quad-\left\langle\bar{\sigma}-\sigma^{d},(-\Delta+I)^{-1}\left(\bar{\sigma}-\sigma^{d}\right)\right\rangle_{\left(\left[H^{1}(\Omega)\right]^{\prime}, H^{1}(\Omega)\right)}\right] \mathrm{d} t+\frac{1}{2} \int_{0}^{T} \int_{\Omega}\left(\mathbf{u}^{\theta}-\overline{\mathbf{u}}\right) \cdot\left(\mathbf{u}^{\theta}+\overline{\mathbf{u}}-2 \mathbf{u}^{d}\right) \mathrm{d} x \mathrm{~d} t \\
& \quad+\frac{\beta}{2} \int_{0}^{T} \int_{\Gamma_{\mathrm{in}}}\left[2 \theta w \bar{w}+\theta^{2} w^{2}\right] \mathrm{d} \mathbf{s} \mathrm{d} t+\frac{\beta}{2} \int_{0}^{T} \int_{\partial \Omega}\left[2 \theta \boldsymbol{\xi} \cdot \overline{\boldsymbol{\xi}}+\theta^{2}|\boldsymbol{\xi}|^{2}\right] \mathrm{d} \mathbf{s} \mathrm{d} t \\
& =\frac{1}{2} \int_{0}^{T}\left[\left\langle\sigma^{\theta}-\bar{\sigma},(-\Delta+I)^{-1}\left(\sigma^{\theta}-\sigma^{d}\right)\right\rangle_{\left(\left[H^{1}(\Omega)\right]^{\prime}, H^{1}(\Omega)\right)}+\left\langle\bar{\sigma}-\sigma^{d},(-\Delta+I)^{-1}\left(\sigma^{\theta}-\bar{\sigma}\right)\right\rangle_{\left(\left[H^{1}(\Omega)\right]^{\prime}, H^{1}(\Omega)\right)}\right] \mathrm{d} t \\
& \quad+\frac{1}{2} \int_{0}^{T} \int_{\Omega}\left(\mathbf{u}^{\theta}-\overline{\mathbf{u}}\right) \cdot\left(\mathbf{u}^{\theta}+\overline{\mathbf{u}}-2 \mathbf{u}^{d}\right) \mathrm{d} x \mathrm{~d} t+\frac{\beta}{2} \int_{0}^{T} \int_{\Gamma_{\mathrm{in}}}\left[2 \theta w \bar{w}+\theta^{2} w^{2}\right] \mathrm{d} \mathbf{s} \mathrm{d} t+\frac{\beta}{2} \int_{0}^{T} \int_{\partial \Omega}\left[2 \theta \boldsymbol{\xi} \cdot \overline{\boldsymbol{\xi}}+\theta^{2}|\boldsymbol{\xi}|^{2}\right] \mathrm{d} \mathbf{s} \mathrm{d} t .
\end{aligned}
$$

So using (4.24) we have,

$$
\begin{aligned}
& \frac{\left(F_{1}(\bar{w}+\theta w, \overline{\boldsymbol{\xi}}+\theta \boldsymbol{\xi})-F_{1}(\bar{w}, \overline{\boldsymbol{\xi}})\right)}{\theta}=\frac{1}{2} \int_{0}^{T}\left[\left\langle\sigma^{w, \xi},(-\Delta+I)^{-1}\left(\sigma^{\theta}-\sigma^{d}\right)\right\rangle_{\left(\left[H^{1}(\Omega)\right]^{\prime}, H^{1}(\Omega)\right)}\right. \\
& \left.+\left\langle\bar{\sigma}-\sigma^{d},(-\Delta+I)^{-1} \sigma^{w, \xi}\right\rangle_{\left(\left[H^{1}(\Omega)\right]^{\prime}, H^{1}(\Omega)\right)}\right] \mathrm{d} t+\frac{1}{2} \int_{0}^{T} \int_{\Omega} \mathbf{u}^{w, \xi} \cdot\left(\mathbf{u}^{\theta}+\overline{\mathbf{u}}-2 \mathbf{u}^{d}\right) \mathrm{d} x \mathrm{~d} t \\
& +\frac{\theta \beta}{2} \int_{0}^{T} \int_{\Gamma_{\mathrm{in}}} w^{2} \mathrm{~d} \mathbf{s} \mathrm{d} t+\beta \int_{0}^{T} \int_{\Gamma_{\mathrm{in}}} w \bar{w} \mathrm{~d} \mathbf{s} \mathrm{d} t+\frac{\theta \beta}{2} \int_{0}^{T} \int_{\partial \Omega}|\boldsymbol{\xi}|^{2} \mathrm{~d} \mathbf{s} \mathrm{d} t+\beta \int_{0}^{T} \int_{\partial \Omega} \boldsymbol{\xi} \cdot \overline{\boldsymbol{\xi}} \mathrm{d} \mathbf{s} \mathrm{d} t .
\end{aligned}
$$


Now taking limit as $\theta \longrightarrow 0$ and using (4.23) we get

$$
\begin{aligned}
\left\langle F_{1}^{\prime}(\bar{w}, \overline{\boldsymbol{\xi}}),(w, \boldsymbol{\xi})\right\rangle= & \int_{0}^{T}\left\langle\sigma^{w, \xi},(-\Delta+I)^{-1}\left(\bar{\sigma}-\sigma^{d}\right)\right\rangle_{\left(\left[H^{1}(\Omega)\right]^{\prime}, H^{1}(\Omega)\right)} \mathrm{d} t+\int_{0}^{T} \int_{\Omega} \mathbf{u}^{w, \xi} \cdot\left(\overline{\mathbf{u}}-\mathbf{u}^{d}\right) \mathrm{d} x \mathrm{~d} t \\
& +\beta \int_{0}^{T} \int_{\Gamma_{\mathrm{in}}} w \bar{w} \mathrm{~d} \mathbf{s} \mathrm{d} t+\beta \int_{0}^{T} \int_{\partial \Omega} \boldsymbol{\xi} \cdot \overline{\boldsymbol{\xi}} \mathrm{d} \mathbf{s} \mathrm{d} t,
\end{aligned}
$$

where $\left(\sigma^{w, \xi}, \mathbf{u}^{w, \xi}\right)$ is the solution of the system in $\Omega_{T}$ :

$$
\begin{gathered}
\frac{\partial \sigma}{\partial t}+\operatorname{div}\left(\sigma \mathbf{v}_{s}\right)=-\operatorname{div}\left(q_{s} \mathbf{u}\right), \\
\frac{\partial \mathbf{u}}{\partial t}-\frac{\mu}{q_{s}} \Delta \mathbf{u}-\frac{(\lambda+\mu)}{q_{s}} \nabla(\operatorname{div} \mathbf{u})+\left(\mathbf{v}_{s} \cdot \nabla\right) \mathbf{u}+(\mathbf{u} \cdot \nabla) \mathbf{v}_{s}+\frac{\sigma}{q_{s}}\left(\mathbf{v}_{s} \cdot \nabla\right) \mathbf{v}_{s}+a \gamma q_{s}^{\gamma-2} \nabla \sigma=\frac{\sigma}{q_{s}} \mathbf{f}, \\
\sigma(0, x)=0, \mathbf{u}(0, x)=\mathbf{0} \text { in } \Omega, \\
\sigma(t, x)=w(t, x) \text { on }(0, T) \times \Gamma_{\text {in }}, \quad \mathbf{u}(t, x)=\boldsymbol{\xi}(t, x) \text { on } \Sigma_{T} .
\end{gathered}
$$

To derive an expression for $F_{1}^{\prime}(\bar{w}, \overline{\boldsymbol{\xi}})$ we introduce the adjoint equation in $\Omega_{T}$

$$
\begin{gathered}
-\frac{\partial \psi}{\partial t}-\mathbf{v}_{s} \cdot \nabla \psi=\frac{\left[\mathbf{f}-\left(\mathbf{v}_{s} \cdot \nabla\right) \mathbf{v}_{s}\right]}{q_{s}} \cdot \boldsymbol{\phi}+a \gamma \operatorname{div}\left(q_{s}^{\gamma-2} \boldsymbol{\phi}\right)+(-\Delta+I)^{-1}\left(\bar{\sigma}-\sigma^{d}\right), \\
-\frac{\partial \phi}{\partial t}-\mu \triangle\left(\frac{\phi}{q_{s}}\right)-(\lambda+\mu) \nabla\left[\operatorname{div}\left(\frac{\phi}{q_{s}}\right)\right]-\left(\operatorname{div}\left(\phi_{1} \mathbf{v}_{s}\right), \operatorname{div}\left(\phi_{2} \mathbf{v}_{s}\right)\right)+\left(\nabla \mathbf{v}_{s}\right)^{T} \boldsymbol{\phi}=q_{s} \nabla \psi+\left(\overline{\mathbf{u}}-\mathbf{u}^{d}\right), \\
\psi(T, x)=0, \phi(T, x)=\mathbf{0} \text { in } \Omega, \\
\psi(t, x)=0 \text { on }(0, T) \times \Gamma_{\text {out }}, \quad \phi(t, x)=\mathbf{0} \text { on } \Sigma_{T} .
\end{gathered}
$$

With formula (4.10) applied to $(\psi, \phi)$ and $\left(\sigma^{w, \xi}, \mathbf{u}^{w, \xi}\right)$ we have

$$
\begin{aligned}
& \int_{0}^{T}\left\langle\sigma^{w, \xi},(-\Delta+I)^{-1}\left(\bar{\sigma}-\sigma^{d}\right)\right\rangle_{\left(\left[H^{1}(\Omega)\right]^{\prime}, H^{1}(\Omega)\right)} \mathrm{d} t+\int_{0}^{T} \int_{\Omega} \mathbf{u}^{w, \xi} \cdot\left(\overline{\mathbf{u}}-\mathbf{u}^{d}\right) \mathrm{d} x \mathrm{~d} t \\
= & \int_{0}^{T} \int_{\Gamma_{\mathrm{in}}} w\left\{v_{s 1} \psi\right\} \mathrm{d} \mathbf{s} \mathrm{d} t-\int_{0}^{T} \int_{\partial \Omega} \boldsymbol{\xi} \cdot\left\{\mu\left[\frac{\partial}{\partial \mathbf{n}}\left(\frac{\phi}{q_{s}}\right)\right]+(\lambda+\mu)\left[\operatorname{div}\left(\frac{\phi}{q_{s}}\right)\right] \mathbf{n}\right\} \mathrm{d} \mathbf{s} \mathrm{d} t .
\end{aligned}
$$

So

$$
\left\langle F_{1}^{\prime}(\bar{w}, \overline{\boldsymbol{\xi}}),(w, \boldsymbol{\xi})\right\rangle=\int_{0}^{T} \int_{\Gamma_{\mathrm{in}}} w\left[\beta \bar{w}+v_{s 1} \psi\right] \mathrm{d} \mathbf{s} \mathrm{d} t-\int_{0}^{T} \int_{\partial \Omega} \boldsymbol{\xi} \cdot\left\{\beta \overline{\boldsymbol{\xi}}+\mu\left[\frac{\partial}{\partial \mathbf{n}}\left(\frac{\phi}{q_{s}}\right)\right]+(\lambda+\mu)\left[\operatorname{div}\left(\frac{\phi}{q_{s}}\right)\right] \mathbf{n}\right\} \mathrm{d} \mathbf{s} \mathrm{d} t .
$$

Hence

$$
F_{1}^{\prime}(\bar{w}, \overline{\boldsymbol{\xi}})=\left(\beta \bar{w}+\left.\left\{v_{s 1} \psi\right\}\right|_{(0, T) \times \Gamma_{\mathrm{in}}}, \beta \overline{\boldsymbol{\xi}}+\mu\left[\frac{\partial}{\partial \mathbf{n}}\left(\frac{\phi}{q_{s}}\right)\right]+(\lambda+\mu)\left[\operatorname{div}\left(\frac{\phi}{q_{s}}\right)\right] \mathbf{n}\right),
$$

where $(\psi, \phi)$ is the solution of adjoint system (2.1)-(2.4) corresponding to

$$
F=(-\Delta+I)^{-1}\left(\bar{\sigma}-\sigma^{d}\right), \quad \mathbf{G}=\overline{\mathbf{u}}-\mathbf{u}^{d} .
$$


Since $(\bar{w}, \overline{\boldsymbol{\xi}})$ is the minimum of $F_{1}, F_{1}^{\prime}(\bar{w}, \overline{\boldsymbol{\xi}})=0$. Hence

$$
\bar{w}=-\left.\frac{1}{\beta}\left\{v_{s 1} \psi\right\}\right|_{(0, T) \times \Gamma_{\text {in }}}, \quad \overline{\boldsymbol{\xi}}=-\frac{1}{\beta}\left\{\mu\left[\frac{\partial}{\partial \mathbf{n}}\left(\frac{\phi}{q_{s}}\right)\right]+(\lambda+\mu)\left[\operatorname{div}\left(\frac{\phi}{q_{s}}\right)\right] \mathbf{n}\right\},
$$

where $(\psi, \phi)$ is the solution of the adjoint system $(2.1)-(2.4)$ corresponding to $F=(-\Delta+I)^{-1}\left(\bar{\sigma}-\sigma^{d}\right), \quad \mathbf{G}=$ $\overline{\mathbf{u}}-\mathbf{u}^{d}$.

Next we derive the sufficient optimality conditions. Due to the previous calculations, we have for every $(\hat{w}, \hat{\boldsymbol{\xi}}) \in L^{2}\left(0, T ; L^{2}\left(\Gamma_{\mathrm{in}}\right)\right) \times L^{2}\left(0, T ; \mathbf{L}^{2}(\partial \Omega)\right)$,

$$
F_{1}^{\prime}(\hat{w}, \hat{\boldsymbol{\xi}})=\left(\beta \hat{w}+\left.\left\{v_{s 1} \hat{\psi}\right\}\right|_{(0, T) \times \Gamma_{\text {in }}}, \beta \hat{\boldsymbol{\xi}}+\mu\left[\frac{\partial}{\partial \mathbf{n}}\left(\frac{\hat{\boldsymbol{\phi}}}{q_{s}}\right)\right]+(\lambda+\mu)\left[\operatorname{div}\left(\frac{\hat{\boldsymbol{\phi}}}{q_{s}}\right)\right] \mathbf{n}\right),
$$

where $(\hat{\psi}, \hat{\phi})$ is the solution of the following system in $\Omega_{T}$

$$
\begin{gathered}
-\frac{\partial \hat{\psi}}{\partial t}-\mathbf{v}_{s} \cdot \nabla \hat{\psi}=\frac{\left[\mathbf{f}-\left(\mathbf{v}_{s} \cdot \nabla\right) \mathbf{v}_{s}\right]}{q_{s}} \cdot \hat{\boldsymbol{\phi}}+a \gamma \operatorname{div}\left(q_{s}^{\gamma-2} \hat{\boldsymbol{\phi}}\right)+(-\Delta+I)^{-1}\left(\hat{\sigma}-\sigma^{d}\right), \\
-\frac{\partial \hat{\boldsymbol{\phi}}}{\partial t}-\mu \triangle\left(\frac{\hat{\boldsymbol{\phi}}}{q_{s}}\right)-(\lambda+\mu) \nabla\left[\operatorname{div}\left(\frac{\hat{\boldsymbol{\phi}}}{q_{s}}\right)\right]-\left(\operatorname{div}\left(\hat{\phi}_{1} \mathbf{v}_{s}\right), \operatorname{div}\left(\hat{\phi}_{2} \mathbf{v}_{s}\right)\right)+\left(\nabla \mathbf{v}_{s}\right)^{T} \hat{\boldsymbol{\phi}}=q_{s} \nabla \hat{\psi}+\left(\hat{\mathbf{u}}-\mathbf{u}^{d}\right), \\
\hat{\psi}(T, x)=0 \text { in } \Omega, \quad \hat{\boldsymbol{\phi}}(T, x)=\mathbf{0} \text { in } \Omega, \\
\hat{\psi}(t, x)=0 \text { on }(0, T) \times \Gamma_{\text {out }}, \quad \hat{\boldsymbol{\phi}}(t, x)=\mathbf{0} \text { on } \Sigma_{T}
\end{gathered}
$$

and $H\left(\sigma, \mathbf{u}_{\mathbf{0}}, \hat{w}, \hat{\boldsymbol{\xi}}\right)=(\hat{\sigma}, \hat{\mathbf{u}})$.

Thus if $((\tilde{\sigma}, \tilde{\mathbf{u}}) ;(\tilde{\psi}, \tilde{\boldsymbol{\phi}}))$ satisfies system $(4.13)-(4.21)$, we have $F_{1}^{\prime}(\complement)=0$, where

$$
\complement=\left(-\left.\frac{1}{\beta}\left\{v_{s 1} \tilde{\psi}\right\}\right|_{(0, T) \times \Gamma_{\mathrm{in}}},-\frac{1}{\beta}\left\{\mu\left[\frac{\partial}{\partial \mathbf{n}}\left(\frac{\tilde{\boldsymbol{\phi}}}{q_{s}}\right)\right]+(\lambda+\mu)\left[\operatorname{div}\left(\frac{\tilde{\boldsymbol{\phi}}}{q_{s}}\right)\right] \mathbf{n}\right\}\right) .
$$

Hence the convexity of the functional $(w, \boldsymbol{\xi}) \longmapsto F_{1}(w, \boldsymbol{\xi})$ implies that

$$
-\frac{1}{\beta}\left(\left.\left\{v_{s 1} \tilde{\psi}\right\}\right|_{(0, T) \times \Gamma_{\mathrm{in}}}, \mu\left[\frac{\partial}{\partial \mathbf{n}}\left(\frac{\tilde{\phi}}{q_{s}}\right)\right]+(\lambda+\mu)\left[\operatorname{div}\left(\frac{\tilde{\phi}}{q_{s}}\right)\right] \mathbf{n}\right)
$$

is the optimal control for problem $(\mathrm{P})$.

Corollary 4.5. From (4.11) and (4.12), using Theorems 2.11 and 2.12, we see that optimal control for problem $(P)$ are more regular, in fact $(\bar{w}, \overline{\boldsymbol{\xi}}) \in L^{2}\left(0, T ; H^{\frac{1}{2}}\left(\Gamma_{\mathrm{in}}\right)\right) \times L^{2}\left(0, T ; \mathbf{H}^{\frac{1}{2}}(\partial \Omega)\right)$.

\section{Appendix A}

Here we prove Theorem 2.6 and then established the trace result for the adjoint continuity equation which is mentioned in Remark 3.8. 


\section{A.1 Proof of Theorem 2.6}

Proof. Our target is to prove here the $H^{1}$ regularity of the solution $\check{\psi}$ of $(2.5)$ when the force term $\varphi$ is in $H^{1}$, by getting the estimates for the derivatives of $\bar{\psi}$. For this we first choose a smooth force term $\varphi$ from a dense class in $H^{1}$ and use the representation formula to get an estimate for the derivative of $\check{\psi}$ on $D_{1}$ and $D_{2}$. The main tool used for this estimate is the change of variable formula. After this, using a density argument we complete the proof of $H^{1}$ regularity and finally show that the solution is unique in $H^{1}$.

\section{Existence of $H^{1}$ solution:}

Case 1. Let $\varphi \in C_{c}^{\infty}\left(0, T ; C^{\infty}(\bar{\Omega})\right)$. This class is dense in $L^{2}\left(0, T ; H^{1}(\Omega)\right)$. For this $\varphi$, using Proposition 2.4 we want to show the following estimates:

$$
\begin{aligned}
\max _{[0, T]}\left\|\frac{\partial \breve{\psi}}{\partial x_{i}}(t)\right\|_{L^{2}(\Omega)} \leq C\left(\mathbf{v}_{s}, T, \Omega\right)\|\varphi\|_{L^{2}\left(0, T ; H^{1}(\Omega)\right)} \\
\left\|\frac{\partial \tilde{\psi}}{\partial t}\right\|_{L^{2}\left(0, T ; L^{2}(\Omega)\right)} \leq C\left(\mathbf{v}_{s}, T, \Omega\right)\|\varphi\|_{L^{2}\left(0, T ; H^{1}(\Omega)\right)}
\end{aligned}
$$

Now let us estimate the derivatives of $\check{\psi}$ w.r.t space and time variable. From (2.14) we get on $D_{1}$

$$
\left|\frac{\partial \check{\psi}}{\partial x_{i}}(t, x)\right| \leq \sum_{j=1}^{2}\left[\int_{0}^{t}\left|\frac{\partial \varphi}{\partial X_{j}}(s, \mathbf{X}(s, t, x))\right|\left|\frac{\partial X_{j}}{\partial x_{i}}(s, t, x)\right| \mathrm{d} s\right] .
$$

Since $X_{1}$ and $X_{2}$ are $C^{2}$ functions, their derivative will be bounded on $[0, T] \times \bar{\Omega}$. Therefore from the above, using Hölder's inequality

$$
\left|\frac{\partial \check{\psi}}{\partial x_{i}}(t, x)\right| \leq C_{1}\left(\mathbf{v}_{s}, T, \Omega\right) \sqrt{T} \sum_{j=1}^{2}\left\{\int_{0}^{t}\left|\frac{\partial \varphi}{\partial X_{j}}(s, \mathbf{X}(s, t, x))\right|^{2} \mathrm{~d} s\right\}^{\frac{1}{2}}
$$

and hence

$$
\int_{D_{1}^{t}}\left|\frac{\partial \check{\psi}}{\partial x_{i}}(t, x)\right|^{2} \mathrm{~d} x \leq C_{2}\left(\mathbf{v}_{s}, T, \Omega\right) \sum_{j=1}^{2} \int_{D_{1}^{t}} \int_{0}^{t}\left|\frac{\partial \varphi}{\partial X_{j}}(s, \mathbf{X}(s, t, x))\right|^{2} \mathrm{~d} s \mathrm{~d} x
$$

where

$$
D_{1}^{t}=\left\{x \in \Omega:(t, x) \in D_{1}\right\} .
$$

Now for estimating the R.H.S of (A.3), we will use the change of variable formula. For that let us define the $\operatorname{map} \beta_{1}^{t}:(0, t) \times D_{1}^{t} \longrightarrow U_{1}$ by

$$
\beta_{1}^{t}(s, x)=(s, \mathbf{X}(s, t, x))=\left(z_{1}, z_{2}, z_{3}\right),
$$

where $U_{1}=\beta_{1}^{t}\left\{(0, t) \times D_{1}^{t}\right\}$ and show that it is a diffeomorphism.

Since $\mathbf{X}(s, t, x)$ is the unique $C^{2}$ solution of the O.D.E:

$$
\frac{\mathrm{d} \mathbf{X}}{\mathrm{d} s}=-\mathbf{v}_{s}(\mathbf{X}), \quad \mathbf{X}(s, t, x)=x \text { for } s=t,
$$

$\beta_{1}^{t}$ is bijective, $C^{1}$ and the Jacobian matrix of $\beta_{1}^{t}$ is

$$
D \beta_{1}^{t}(s, x)=\left[\begin{array}{ccc}
1 & 0 & 0 \\
\frac{\partial X_{1}}{\partial s}(s, t, x) & \frac{\partial X_{1}}{\partial x_{1}}(s, t, x) & \frac{\partial X_{1}}{\partial x_{2}}(s, t, x) \\
\frac{\partial X_{2}}{\partial s}(s, t, x) & \frac{\partial X_{2}}{\partial x_{1}}(s, t, x) & \frac{\partial X_{2}}{\partial x_{2}}(s, t, x)
\end{array}\right] .
$$


Using Girinon [5] (Chap. IV, Sect. 2.1), we have

$$
\left|\begin{array}{l}
\frac{\partial X_{1}}{\partial x_{1}}(s, t, x) \frac{\partial X_{1}}{\partial x_{2}}(s, t, x) \\
\frac{\partial X_{2}}{\partial x_{1}}(s, t, x) \frac{\partial X_{2}}{\partial x_{2}}(s, t, x)
\end{array}\right|=\exp \left(\int_{0}^{s-t} \operatorname{div}_{s}(\mathbf{X}(r, 0, x)) \mathrm{d} r\right)
$$

and hence $\operatorname{det}\left[D \beta_{1}^{t}(s, x)\right]>0$. Therefore, $\beta_{1}^{t}$ is a diffeomorphism. Let

$$
M=\sup _{p \in \mathbb{R}^{2}}\left|\operatorname{div} \mathbf{v}_{s}(p)\right| .
$$

As $0<s<t \leq T$,

$$
-\int_{s-t}^{0} \operatorname{div}_{s}(\mathbf{X}(r, 0, x)) \mathrm{d} r \geq-M(t-s)
$$

Then for $(s, x) \in(0, t) \times D_{1}^{t}$,

$$
\operatorname{det}\left[D \beta_{1}^{t}(s, x)\right]=\exp \left(\int_{0}^{s-t} \operatorname{divv}_{s}(\mathbf{X}(r, 0, x)) \mathrm{d} r\right) \geq \exp (-M T)
$$

and so

$$
\frac{1}{\left|\operatorname{det}\left[D \beta_{1}^{t}(s, x)\right]\right|} \leq \exp (M T)
$$

Now using (A.5)

$$
\begin{aligned}
\int_{D_{1}^{t}} \int_{0}^{t}\left|\frac{\partial \varphi}{\partial X_{1}}(s, \mathbf{X}(s, t, x))\right|^{2} \mathrm{~d} s \mathrm{~d} x & =\int_{D_{1}^{t}} \int_{0}^{t} \frac{1}{\left|\operatorname{det}\left[D \beta_{1}^{t}(s, x)\right]\right|}\left|\frac{\partial \varphi}{\partial X_{1}}\left(\beta_{1}^{t}(s, x)\right)\right|^{2}\left|\operatorname{det}\left[D \beta_{1}^{t}(s, x)\right]\right| \mathrm{d} s \mathrm{~d} x \\
& \leq \exp (M T) \int_{U_{1}}\left|\frac{\partial \varphi}{\partial z_{2}}\left(z_{1}, z_{2}, z_{3}\right)\right|^{2} \mathrm{~d} z_{1} \mathrm{~d} z_{2} \mathrm{~d} z_{3} \\
& \leq \exp (M T) \int_{0}^{T} \int_{\Omega}\left|\frac{\partial \varphi}{\partial z_{2}}\left(z_{1}, z_{2}, z_{3}\right)\right|^{2} \mathrm{~d} z_{1} \mathrm{~d} z_{2} \mathrm{~d} z_{3} .
\end{aligned}
$$

Thus we get for $j=1,2$

$$
\int_{D_{1}^{t}} \int_{0}^{t}\left|\frac{\partial \varphi}{\partial X_{j}}(s, \mathbf{X}(s, t, x))\right|^{2} \mathrm{~d} s \mathrm{~d} x \leq \exp (M T)\|\varphi\|_{L^{2}\left(0, T ; H^{1}(\Omega)\right)}^{2} .
$$

Hence combining (A.6) with (A.3), we get on $D_{1}$

$$
\int_{D_{1}^{t}}\left|\frac{\partial \check{\psi}}{\partial x_{i}}(t, x)\right|^{2} \mathrm{~d} x \leq C\left(\mathbf{v}_{s}, T, \Omega\right)\|\varphi\|_{L^{2}\left(0, T ; H^{1}(\Omega)\right)}^{2} .
$$

Also from (2.15), for a similar estimate on $D_{2}$, we begin with

$$
\int_{D_{2}^{t}} \int_{t_{2}(t, x)}^{t}\left|\frac{\partial \varphi}{\partial X_{j}}(s, \mathbf{X}(s, t, x))\right|^{2} \mathrm{~d} s \mathrm{~d} x
$$

where $D_{2}^{t}=\left\{x \in \Omega:(t, x) \in D_{2}\right\}$. Since the interval $\left(t_{2}(t, x), t\right)$ changes w.r.to $x$, we cannot directly apply the change of variable formula and estimate as before. So we go to a bigger interval $(0, t)$ which does not vary with $x$ and estimate using change of variable as on $D_{1}$. But notice that for $(t, x) \in D_{2},\left(t_{2}, \mathbf{X}\left(t_{2}, t, x\right)\right)$ is a point 
on the wall $\left\{x_{1}=1\right\}$ and $(0, \mathbf{X}(0, t, x))$ is outside of $(0, t) \times(0,1) \times(0, h)$ where $\varphi$ is not defined. So we use a continuous $H^{1}$ extension $\tilde{\varphi} \in L^{2}\left(0, T ; H^{1}\left(\mathbb{R}^{2}\right)\right)$ of $\varphi \in L^{2}\left(0, T ; H^{1}(\Omega)\right)$ and get for $j=1,2$

$$
\int_{D_{2}^{t}} \int_{t_{2}(t, x)}^{t}\left|\frac{\partial \varphi}{\partial X_{j}}(s, \mathbf{X}(s, t, x))\right|^{2} \mathrm{~d} s \mathrm{~d} x \leq \int_{D_{2}^{t}} \int_{0}^{t}\left|\frac{\partial \tilde{\varphi}}{\partial X_{j}}(s, \mathbf{X}(s, t, x))\right|^{2} \mathrm{~d} s \mathrm{~d} x .
$$

Since $\Omega$ is a bounded domain with Lipschitz boundary, we know from Sobolev space theory that such continuous $H^{1}$ extension exists. For details see [3] (Chap. 4, Sect. 4.4, Thm. 1).

Now we will consider the change of variable $\beta_{2}^{t}:(0, t) \times D_{2}^{t} \longrightarrow U_{2}=\beta_{2}^{t}\left\{(0, t) \times D_{2}^{t}\right\}$ defined by

$$
\beta_{2}^{t}(s, x)=(s, \mathbf{X}(s, t, x))=\left(z_{1}, z_{2}, z_{3}\right)
$$

and estimate the R.H.S of (A.8). Using $\frac{1}{\left|\operatorname{det}\left[D \beta_{2}^{t}(s, x)\right]\right|} \leq \exp (M T)$ and the continuity of the extension operator we get

$$
\begin{aligned}
\int_{D_{2}^{t}} \int_{0}^{t}\left|\frac{\partial \tilde{\varphi}}{\partial X_{1}}(s, \mathbf{X}(s, t, x))\right|^{2} \mathrm{~d} s \mathrm{~d} x & \leq \exp (M T) \int_{U_{2}}\left|\frac{\partial \tilde{\varphi}}{\partial z_{2}}\left(z_{1}, z_{2}, z_{3}\right)\right|^{2} \mathrm{~d} z_{1} \mathrm{~d} z_{2} \mathrm{~d} z_{3} \\
& \leq \exp (M T) \int_{0}^{T} \int_{\mathbb{R}^{2}}\left|\frac{\partial \tilde{\varphi}}{\partial z_{2}}\left(z_{1}, z_{2}, z_{3}\right)\right|^{2} \mathrm{~d} z_{1} \mathrm{~d} z_{2} \mathrm{~d} z_{3} \\
& \leq \exp (M T)\|\tilde{\varphi}\|_{L^{2}\left(0, T ; H^{1}\left(\mathbb{R}^{2}\right)\right)}^{2} \leq C\left(\mathbf{v}_{s}, T, \Omega\right)\|\varphi\|_{L^{2}\left(0, T ; H^{1}(\Omega)\right)}^{2} .
\end{aligned}
$$

So from (A.8), we get for $j=1,2$

$$
\int_{D_{2}^{t}} \int_{t_{2}(t, x)}^{t}\left|\frac{\partial \varphi}{\partial X_{j}}(s, \mathbf{X}(s, t, x))\right|^{2} \mathrm{~d} s \mathrm{~d} x \leq C\left(\mathbf{v}_{s}, T, \Omega\right)\|\varphi\|_{L^{2}\left(0, T ; H^{1}(\Omega)\right)}^{2} .
$$

Now we want to estimate:

$$
\int_{D_{2}^{t}}\left|\frac{\partial t_{2}}{\partial x_{i}}(t, x)\right|^{2}\left|\varphi\left(t_{2}(t, x), 1, X_{2}\left(t_{2}(t, x), t, x\right)\right)\right|^{2} \mathrm{~d} x
$$

We define a map $K^{t}: D_{2}^{t} \longrightarrow K^{t}\left(D_{2}^{t}\right)$,

$$
K^{t}(x)=\left(K_{1}^{t}(x), K_{2}^{t}(x)\right)=\left(t_{2}(t, x), X_{2}\left(t_{2}(t, x), t, x\right)\right)=\left(y_{1}, y_{3}\right) .
$$

Clearly $K^{t}$ is bijective and $C^{1}$.

$$
\begin{gathered}
D K^{t}(x)=\left[\begin{array}{c}
\frac{\partial t_{2}}{\partial x_{1}}(t, x) \\
\frac{\partial t_{2}}{\partial x_{2}}(t, x) \\
\frac{\partial X_{2}}{\partial t_{2}}\left(t_{2}, t, x\right) \frac{\partial t_{2}}{\partial x_{1}}(t, x)+\frac{\partial X_{2}}{\partial x_{1}}\left(t_{2}, t, x\right) \frac{\partial X_{2}}{\partial t_{2}}\left(t_{2}, t, x\right) \frac{\partial t_{2}}{\partial x_{2}}(t, x)+\frac{\partial X_{2}}{\partial x_{2}}\left(t_{2}, t, x\right)
\end{array}\right], \\
\Rightarrow \operatorname{det}\left[D K^{t}(x)\right]=\frac{\partial t_{2}}{\partial x_{1}}(t, x) \frac{\partial X_{2}}{\partial x_{2}}\left(t_{2}, t, x\right)-\frac{\partial t_{2}}{\partial x_{2}}(t, x) \frac{\partial X_{2}}{\partial x_{1}}\left(t_{2}, t, x\right) \\
=\frac{1}{v_{s 1}\left(X\left(t_{2}, t, x\right)\right)}\left|\frac{\partial X_{1}}{\partial x_{2}}\left(t_{2}, t, x\right) \frac{\partial X_{1}}{\partial x_{1}}\left(t_{2}, t, x\right) \frac{\partial X_{2}}{\partial x_{2}}\left(t_{2}, t, x\right)\right|[\operatorname{using}(2.10)] \\
=\frac{1}{v_{s 1}\left(X\left(t_{2}, t, x\right)\right)} \exp \left(\int_{0}^{t_{2}-t} \operatorname{div}_{s}(\mathbf{X}(r, 0, x)) \mathrm{d} r\right) \neq 0
\end{gathered}
$$


Hence $K^{t}$ is a diffeomorphism. Also from (A.10), using $v_{s 1} \geq \alpha>0$ on $\overline{\Gamma_{\text {out }}}$ and (A.4) we get

$$
\frac{1}{\left|\operatorname{det}\left[D K^{t}(x)\right]\right|} \leq \frac{\exp (M T)}{\alpha}
$$

So, using (2.11), (A.11) and the continuity of the trace map

$$
\begin{aligned}
& \int_{D_{2}^{t}}\left|\frac{\partial t_{2}}{\partial x_{i}}(t, x)\right|^{2}\left|\varphi\left(t_{2}(t, x), 1, X_{2}\left(t_{2}(t, x), t, x\right)\right)\right|^{2} \mathrm{~d} x \\
\leq & C_{1}\left(\mathbf{v}_{s}, T, \Omega\right) \int_{D_{2}^{t}}\left|\varphi\left(t_{2}(t, x), 1, X_{2}\left(t_{2}(t, x), t, x\right)\right)\right|^{2} \mathrm{~d} x \\
= & C_{1}\left(\mathbf{v}_{s}, T, \Omega\right) \int_{D_{2}^{t}} \frac{1}{\left|\operatorname{det}\left[D K^{t}(x)\right]\right|} \varphi^{2}\left(K_{1}^{t}(x), 1, K_{2}^{t}(x)\right)\left|\operatorname{det}\left[D K^{t}(x)\right]\right| \mathrm{d} x \\
\leq & C_{2}\left(\mathbf{v}_{s}, T, \Omega\right) \int_{K^{t}\left(D_{2}^{t}\right)} \varphi^{2}\left(y_{1}, 1, y_{3}\right) \mathrm{d} y_{1} \mathrm{~d} y_{3} \\
\leq & C_{2}\left(\mathbf{v}_{s}, T, \Omega\right) \int_{0}^{T}\left[\int_{0}^{h} \varphi^{2}\left(y_{1}, 1, y_{3}\right) \mathrm{d} y_{3}\right] \mathrm{d} y_{1} \\
\leq & C\left(\mathbf{v}_{s}, T, \Omega\right) \int_{0}^{T}\left\|\varphi\left(y_{1}\right)\right\|_{H^{1}(\Omega)}^{2} \mathrm{~d} y_{1}=C\left(\mathbf{v}_{s}, T, \Omega\right)\|\varphi\|_{L^{2}\left(0, T ; H^{1}(\Omega)\right)}^{2} .
\end{aligned}
$$

Hence using (A.9) and (A.12) from (2.15) we get on $D_{2}$

$$
\begin{aligned}
\int_{D_{2}^{t}}\left|\frac{\partial \breve{\psi}}{\partial x_{i}}(t, x)\right|^{2} \mathrm{~d} x \leq & C_{2}\left(\mathbf{v}_{s}, T, \Omega\right) \sum_{j=1}^{2} \int_{D_{2}^{t}} \int_{t_{2}(t, x)}^{t}\left|\frac{\partial \varphi}{\partial X_{j}}(s, \mathbf{X}(s, t, x))\right|^{2} \mathrm{~d} s \mathrm{~d} x \\
& +2 \int_{D_{2}^{t}}\left|\frac{\partial t_{2}}{\partial x_{i}}(t, x)\right|^{2}\left|\varphi\left(t_{2}(t, x), 1, X_{2}\left(t_{2}(t, x), t, x\right)\right)\right|^{2} \mathrm{~d} x \\
\leq & C\left(\mathbf{v}_{s}, T, \Omega\right)\|\varphi\|_{L^{2}\left(0, T ; H^{1}(\Omega)\right)}^{2} .
\end{aligned}
$$

Let us now estimate the time derivative. From (2.14) we get on $D_{1}$

$$
\left|\frac{\partial \breve{\psi}}{\partial t}(t, x)\right| \leq \sum_{j=1}^{2} \int_{0}^{t}\left|\frac{\partial \varphi}{\partial X_{j}}(s, \mathbf{X}(s, t, x))\right|\left|\frac{\partial X_{j}}{\partial t}(s, t, x)\right| \mathrm{d} s+|\varphi(t, x)| .
$$

Thus

$$
\left|\frac{\partial \check{\psi}}{\partial t}(t, x)\right| \leq C_{1}\left(\mathbf{v}_{s}, T, \Omega\right) \sqrt{T} \sum_{j=1}^{2}\left\{\int_{0}^{t}\left|\frac{\partial \varphi}{\partial X_{j}}(s, \mathbf{X}(s, t, x))\right|^{2} \mathrm{~d} s\right\}^{\frac{1}{2}}+|\varphi(t, x)| .
$$

So,

$$
\int_{D_{1}^{t}}\left|\frac{\partial \breve{\psi}}{\partial t}(t, x)\right|^{2} \mathrm{~d} x \leq C_{2}\left(\mathbf{v}_{s}, T, \Omega\right) \sum_{j=1}^{2} \int_{D_{1}^{t}} \int_{0}^{t}\left|\frac{\partial \varphi}{\partial X_{j}}(s, \mathbf{X}(s, t, x))\right|^{2} \mathrm{~d} s \mathrm{~d} x+2 \int_{D_{1}^{t}}|\varphi(t, x)|^{2} \mathrm{~d} x .
$$

Using (A.6) we get from above

$$
\int_{0}^{T} \int_{D_{1}^{t}}\left|\frac{\partial \check{\psi}}{\partial t}(t, x)\right|^{2} \mathrm{~d} x \mathrm{~d} t \leq T C_{3}\left(\mathbf{v}_{s}, T, \Omega\right)\|\varphi\|_{L^{2}\left(0, T ; H^{1}(\Omega)\right)}^{2}+2\|\varphi\|_{L^{2}\left(0, T ; L^{2}(\Omega)\right)}^{2} .
$$


Therefore, on $D_{1}$

$$
\left\|\frac{\partial \check{\psi}}{\partial t}\right\|_{L^{2}\left(D_{1}\right)} \leq C\left(\mathbf{v}_{s}, T, \Omega\right)\|\varphi\|_{L^{2}\left(0, T ; H^{1}(\Omega)\right)}
$$

and on $D_{2}$,

$$
\begin{aligned}
\int_{D_{2}^{t}}\left|\frac{\partial \check{\psi}}{\partial t}(t, x)\right|^{2} \mathrm{~d} x \leq & C_{2}\left(\mathbf{v}_{s}, T, \Omega\right) \sum_{j=1}^{2} \int_{D_{2}^{t}} \int_{t_{2}(t, x)}^{t}\left|\frac{\partial \varphi}{\partial X_{j}}(s, \mathbf{X}(s, t, x))\right|^{2} \mathrm{~d} s \mathrm{~d} x+3 \int_{D_{2}^{t}}|\varphi(t, x)|^{2} \mathrm{~d} x \\
& +3 \int_{D_{2}^{t}}\left|\frac{\partial t_{2}}{\partial t}(t, x)\right|^{2}\left|\varphi\left(t_{2}(t, x), 1, X_{2}\left(t_{2}(t, x), t, x\right)\right)\right|^{2} \mathrm{~d} x .
\end{aligned}
$$

Hence using (2.11), (A.12) and (A.9) we get on $D_{2}$ :

$$
\left\|\frac{\partial \check{\psi}}{\partial t}\right\|_{L^{2}\left(D_{2}\right)} \leq C\left(\mathbf{v}_{s}, T, \Omega\right)\|\varphi\|_{L^{2}\left(0, T ; H^{1}(\Omega)\right)} .
$$

Using the change of variables $\beta_{1}^{t}, \beta_{2}^{t}$ and following the same estimation of derivative from representation formula, we will get the $L^{2}$ estimate of $\tilde{\psi}(t)$

$$
\max _{[0, T]}\|\breve{\psi}(t)\|_{L^{2}(\Omega)} \leq C\left(\mathbf{v}_{s}, T, \Omega\right)\|\varphi\|_{L^{2}\left(0, T ; L^{2}(\Omega)\right)} .
$$

For proving the above $L^{2}$ estimate in $D_{2}$, note that we will choose $\tilde{\varphi}$ as zero extension of $\varphi$. Thus proof of (2.18) is completed in this case.

Case 2. Let $\varphi \in L^{2}\left(0, T ; H^{1}(\Omega)\right)$. Since $C_{c}^{\infty}(0, T)$ is dense in $L^{2}(0, T)$ and $C^{\infty}(\bar{\Omega})$ is dense in $H^{1}(\Omega)$, there exists a sequence $\left\{\varphi_{n}\right\}_{\{n \in \mathbb{N}\}} \in C_{c}^{\infty}\left(0, T ; C^{\infty}(\bar{\Omega})\right)$ such that

$$
\varphi_{n} \longrightarrow \varphi \text { in } L^{2}\left(0, T ; H^{1}(\Omega)\right) \text {. }
$$

Let $\check{\psi}_{n}$ be the solution of (2.5) corresponding to $\varphi_{n}$. From (2.18) and expressions (2.13)-(2.15), we see that $\check{\psi}_{n} \in C\left([0, T] ; H^{1}(\Omega)\right)$ is a Cauchy sequence in $L^{\infty}\left(0, T ; H^{1}(\Omega)\right) \cap H^{1}\left(0, T ; L^{2}(\Omega)\right)$ and hence converges to some $\check{\psi}$ in $C\left([0, T] ; H^{1}(\Omega)\right) L^{\infty}\left(0, T ; H^{1}(\Omega)\right) \cap H^{1}\left(0, T ; L^{2}(\Omega)\right) \cap H^{1}\left(0, T ; L^{2}(\Omega)\right)$. Therefore we get a strong solution $\check{\psi} \in C\left([0, T] ; H^{1}(\Omega)\right) \cap H^{1}\left(0, T ; L^{2}(\Omega)\right)$ of $(2.5)$ for $\varphi \in L^{2}\left(0, T ; H^{1}(\Omega)\right)$.

Uniqueness: Now we will show that the solution of $(2.5)$ is unique in the class $L^{2}\left(0, T ; H^{1}(\Omega) \cap H^{1}\left(0, T ; L^{2}(\Omega)\right)\right.$. Let $\check{\psi}_{i} \in L^{2}\left(0, T ; H^{1}(\Omega)\right) \cap H^{1}\left(0, T ; L^{2}(\Omega)\right), i=1,2$ be two solutions of $(2.5)$. Let us denote $\bar{\psi}=\check{\psi}_{1}-\check{\psi}_{2}$. Then $\bar{\psi}$ satisfies

$$
\left.\begin{array}{c}
\frac{\partial \bar{\psi}}{\partial t}(t, x)-\mathbf{v}_{s}(x) \cdot \nabla \bar{\psi}(t, x)=0 \text { in } \Omega_{T}, \\
\bar{\psi}(0, x)=0 \text { in } \Omega, \quad \bar{\psi}(t, x)=0 \text { on }(0, T) \times \Gamma_{\text {out }},
\end{array}\right\}
$$

where (1.8) holds for $\mathbf{v}_{s}$.

Multiplying (A.18) by $\bar{\psi}$, using $v_{s 2}=0$ on $\Gamma_{0}, \bar{\psi}=0$ on $(0, T) \times \Gamma_{\text {out }}, n_{1}=0$ on $\Gamma_{0}, n_{2}=0$ on $\Gamma_{\text {in }}$ where $\mathbf{n}=\left(n_{1}, n_{2}\right)$ denotes unit outward normal to $\partial \Omega$ and doing integration by parts we get

$$
\frac{\mathrm{d}}{\mathrm{d} t}\|\bar{\psi}\|_{L^{2}(\Omega)}^{2}+\int_{\Omega} \operatorname{div}\left(\mathbf{v}_{s}\right) \bar{\psi}^{2} \mathrm{~d} x+\int_{\Gamma_{\mathrm{in}}} v_{s 1} \bar{\psi}^{2} \mathrm{~d} \mathbf{s}=0
$$

Since $v_{s 1} \geq \alpha>0$ on $\overline{\Gamma_{\mathrm{in}}}$,

$$
\frac{\mathrm{d}}{\mathrm{d} t}\|\bar{\psi}\|_{L^{2}(\Omega)}^{2} \leq-\int_{\Omega} \operatorname{div}\left(\mathbf{v}_{s}\right) \bar{\psi}^{2} \mathrm{~d} x .
$$


Thus

$$
\frac{\mathrm{d}}{\mathrm{d} t}\|\bar{\psi}\|_{L^{2}(\Omega)}^{2} \leq\left\|\operatorname{div}\left(\mathbf{v}_{s}\right)\right\|_{L^{\infty}(\Omega)}\|\bar{\psi}\|_{L^{2}(\Omega)}^{2} \text { and }\|\bar{\psi}(0)\|_{L^{2}(\Omega)}=0 .
$$

Hence using Gronwall's inequality, we get $\bar{\psi}=0$ and so $\check{\psi}_{1}=\check{\psi}_{2}$. This completes the proof.

\section{A.2 Trace result for the adjoint continuity equation}

In this section we give the proof of "hidden regularity" mentioned in Remark 3.8 for the adjoint continuity equation. This trace result is required to define the solution of non homogeneous initial-boundary value problem for continuity equation via transposition and to obtain a $L^{2}\left(0, T ; L^{2}(\Omega)\right)$ solution.

Let us consider

$$
\left.\begin{array}{rl}
-\frac{\partial \psi}{\partial t}-\mathbf{v}_{s} \cdot \nabla \psi & =\varphi \text { in } \Omega_{T}, \\
\psi(t, x) & =0 \text { on }(0, T) \times \Gamma_{\text {out }}, \\
\psi(T, x) & =0 \text { in } \Omega,
\end{array}\right\}
$$

where $\varphi \in L^{2}\left(0, T ; L^{2}(\Omega)\right)$ and (1.8) holds for $\mathbf{v}_{s}$. Following theorem, gives the existence of the weak solution for the problem (A.19). The proof uses semigroup approach and can be found in [4] (Thm. I.12, p. 65).

Theorem A.1. For $\varphi \in L^{2}\left(0, T ; L^{2}(\Omega)\right)$, problem (A.19) admits a unique weak solution $\psi \in C\left([0, T] ; L^{2}(\Omega)\right)$ and we have the following estimate

$$
\|\psi(t)\|_{L^{2}(\Omega)} \leq C(T)\|\varphi\|_{L^{2}\left(0, T ; L^{2}(\Omega)\right)}
$$

for some constant $C(T)>0$.

Theorem A.2 (Hidden regularity). If $\varphi \in L^{2}\left(0, T ; L^{2}(\Omega)\right)$, then $\left.\psi\right|_{\Gamma_{\mathrm{in}}} \in L^{2}\left(0, T ; L^{2}\left(\Gamma_{\mathrm{in}}\right)\right)$ and the map $\varphi \rightarrow$ $\left.\psi\right|_{\Gamma_{\mathrm{in}}}$ is continuous from $L^{2}\left(0, T ; L^{2}(\Omega)\right)$ into $L^{2}\left(0, T ; L^{2}\left(\Gamma_{\mathrm{in}}\right)\right)$.

Proof. We will prove this regularity result using multiplier method.

Case 1. Let us consider the case when $\varphi \in L^{2}\left(0, T ; H^{1}(\Omega)\right)$. Then from Theorem 2.6 we get $\psi$ belongs to $H^{1}\left(0, T ; L^{2}(\Omega)\right) \cap C\left([0, T] ; H^{1}(\Omega)\right)$. Now multiplying (A.19) by $\psi$ and integrating over $(0, T) \times \Omega$ we get

$$
-\int_{0}^{T} \int_{\Omega} \frac{\partial \psi}{\partial t} \psi \mathrm{d} x \mathrm{~d} t-\int_{0}^{T} \int_{\Omega}\left(\mathbf{v}_{s} \cdot \nabla \psi\right) \psi \mathrm{d} x \mathrm{~d} t=\int_{0}^{T} \int_{\Omega} \varphi \psi \mathrm{d} x \mathrm{~d} t .
$$

Using integration by parts we get,

$$
-\frac{1}{2} \int_{\Omega}\left[\psi^{2}(T, x)-\psi^{2}(0, x)\right] \mathrm{d} x+\frac{1}{2} \int_{0}^{T} \int_{\Omega}\left(\operatorname{div} \mathbf{v}_{s}\right) \psi^{2} \mathrm{~d} x \mathrm{~d} t-\frac{1}{2} \int_{0}^{T} \int_{\partial \Omega}\left(\mathbf{v}_{s} \cdot \mathbf{n}\right) \psi^{2} \mathrm{~d} \mathbf{s} \mathrm{d} t=\int_{0}^{T} \int_{\Omega} \varphi \psi \mathrm{d} x \mathrm{~d} t .
$$

Since $\psi(T, x)=0, \mathbf{v}_{s} \cdot \mathbf{n}=0$ on $\Gamma_{0}, \psi=0$ on $\Gamma_{\text {out }}$ and $\mathbf{n}=(-1,0)$ on $\Gamma_{\text {in }}$, we get

$$
\frac{1}{2} \int_{\Omega} \psi^{2}(0, x) \mathrm{d} x+\frac{1}{2} \int_{0}^{T} \int_{\Omega}\left(\operatorname{div} \mathbf{v}_{s}\right) \psi^{2} \mathrm{~d} x \mathrm{~d} t+\frac{1}{2} \int_{0}^{T} \int_{\Gamma_{\mathrm{in}}} v_{s 1} \psi^{2} \mathrm{~d} \mathbf{s} \mathrm{d} t=\int_{0}^{T} \int_{\Omega} \varphi \psi \mathrm{d} x \mathrm{~d} t .
$$

Thus

$$
\int_{0}^{T} \int_{\Gamma_{\mathrm{in}}} v_{s 1} \psi^{2} \mathrm{~d} \mathbf{s} \mathrm{d} t \leq M \int_{0}^{T} \int_{\Omega} \psi^{2} \mathrm{~d} x \mathrm{~d} t+2 \int_{0}^{T} \int_{\Omega}|\varphi||\psi| \mathrm{d} x \mathrm{~d} t,
$$

where $\mathrm{M}$ is given by (A.4). Using $v_{s 1} \geq \alpha>0$ on $\overline{\Gamma_{\mathrm{in}}}$ and Hölders inequality we get

$$
\alpha\left\|\left.\left.\psi\right|_{\Gamma_{\mathrm{in}}}\right|_{L^{2}\left(0, T ; L^{2}\left(\Gamma_{\mathrm{in}}\right)\right)} ^{2} \leq M\right\| \psi\left\|_{L^{2}\left(0, T ; L^{2}(\Omega)\right)}^{2}+2\right\| \varphi\left\|_{L^{2}\left(0, T ; L^{2}(\Omega)\right)}\right\| \psi \|_{L^{2}\left(0, T ; L^{2}(\Omega)\right)} .
$$


So from (A.20) and (A.21) we get

$$
\left\|\left.\psi\right|_{\Gamma_{\mathrm{in}}}\right\|_{L^{2}\left(0, T ; L^{2}\left(\Gamma_{\mathrm{in}}\right)\right)} \leq C\left(T, \mathbf{v}_{s}\right)\|\varphi\|_{L^{2}\left(0, T ; L^{2}(\Omega)\right)} \forall \varphi \in L^{2}\left(0, T ; H^{1}(\Omega)\right) .
$$

Case 2. Let $\varphi \in L^{2}\left(0, T ; L^{2}(\Omega)\right)$. So there exists a sequence $\varphi_{n} \in L^{2}\left(0, T ; H^{1}(\Omega)\right)$ such that $\varphi_{n} \longrightarrow \varphi \in$ $L^{2}\left(0, T ; L^{2}(\Omega)\right)$. Let $\psi_{n}$ be the solution of (A.19) corresponding to $\varphi_{n}$. Then according to (A.22) we have

$$
\left\|\left.\psi_{n}\right|_{\Gamma_{\mathrm{in}}}-\left.\left.\psi_{m}\right|_{\Gamma_{\mathrm{in}}}\right|_{L^{2}\left(0, T ; L^{2}\left(\Gamma_{\mathrm{in}}\right)\right)} \leq C|| \varphi_{n}-\varphi_{m}\right\|_{L^{2}\left(0, T ; L^{2}(\Omega)\right)} .
$$

So $\left.\psi_{n}\right|_{\Gamma_{\text {in }}}$ is a Cauchy sequence in $L^{2}\left(0, T ; L^{2}\left(\Gamma_{\mathrm{in}}\right)\right)$. Therefore $\left.\psi_{n}\right|_{\Gamma_{\mathrm{in}}}$ converges in $L^{2}\left(0, T ; L^{2}\left(\Gamma_{\mathrm{in}}\right)\right)$. From $($ A.20) we have $\psi_{n}$ converges to $\psi$ in $C\left([0, T] ; L^{2}(\Omega)\right)$, where $\psi \in C\left([0, T] ; L^{2}(\Omega)\right)$ is the weak solution of (A.19) corresponding to $\varphi$ mentioned in Theorem 5.1. We define

$$
\left.\psi\right|_{\Gamma_{\text {in }}}=\left.\lim _{n \rightarrow \infty} \psi_{n}\right|_{\Gamma_{\text {in }}}
$$

the limit taken in $L^{2}\left(0, T ; L^{2}\left(\Gamma_{\mathrm{in}}\right)\right)$. According to (A.23) this definition does not depend on the particular choice of regular functions approximating $\varphi$. Hence

$$
\left\|\left.\psi\right|_{\Gamma_{\mathrm{in}}}\right\|_{L^{2}\left(0, T ; L^{2}\left(\Gamma_{\mathrm{in}}\right)\right)} \leq C\left(T, \mathbf{v}_{s}\right)\|\varphi\|_{L^{2}\left(0, T ; L^{2}(\Omega)\right)} \forall \varphi \in L^{2}\left(0, T ; L^{2}(\Omega)\right) .
$$

This completes the proof.

Acknowledgements. The authors would like to thank Prof. Jean-Pierre Raymond for useful discussions. The authors acknowledge the financial support from the Indo-French Centre for the Promotion of Advanced Research, Delhi, under project 3701-1.

\section{REFERENCES}

[1] A. Bensoussan, G. Da Prato, M.C. Delfour and S.K. Mitter, Representation and Control of Infinite Dimensional Systems, 2nd edition. Birkhäuser (2006).

[2] R. Dautray and J.-L. Lions, Mathematical analysis and numerical methods for science and technology, in Evolution Problems. I. With the collaboration of M. Artola, M. Cessenat and H. Lanchon. Translated from the French by A. Craig. Springer-Verlag, Berlin 5 (1992).

[3] L.C. Evans and R.F. Gariepy, Measure theory and fine properties of functions. Studies in Advanced Mathematics. CRC Press, Boca Raton, FL (1992).

[4] G. Geymonat and P. Leyland, Transport and propagation of a perturbation of a flow of a compressible fluid in a bounded region. Arch. Rational Mech. Anal. 100 (1987) 53-81.

[5] V. Girinon, Quelques problémes aux limites pour les équations de Navier-Stokes compressibles. Ph.D. thesis, Université de Toulouse (2008).

[6] M.D. Gunzburger and S. Manservisi, The velocity tracking problem for Navier-Stokes flows with boundary control. SIAM J. Control Optim. 39 (2000) 594-634.

[7] V.I. Judovič, A two-dimensional problem of unsteady flow of an ideal incompressible fluid across a given domain. Amer. Math. Soc. Trans. 57 (1966) 277-304 [previously in Mat. Sb. (N.S.) 64 (1964) 562-588 (in Russian)].

[8] J. Neustupa, A semigroup generated by the linearized Navier-Stokes equations for compressible fluid and its uniform growth bound in Hölder spaces. Navier-Stokes equations: theory and numerical methods (Varenna, 1997), Pitman. Research Notes Math. Ser. 388 (1998) 86-100.

[9] J.P. Raymond, Stokes and Navier-Stokes equations with nonhomogeneous boundary conditions. Ann. Inst. Henri Poincaré Anal. Non Linéaire 24 (2007) 921-951.

[10] J.P. Raymond and A.P. Nguyen, Control localized on thin structures for the linearized Boussinesq system. J. Optim. Theory Appl. 141 (2009) 147-165.

[11] A. Valli and W.M. Zajczkowski, Navier-Stokes equations for compressible fluids: global existence and qualitative properties of the solutions in the general case. Commun. Math. Phys. 103 (1986) 259-296. 